Constructing strong linearizations of matrix polynomials expressed in the Chebyshev bases

Lawrence, Piers and Perez, Javier 2016

MIMS EPrint: 2016.12

Manchester Institute for Mathematical Sciences

School of Mathematics

The University of Manchester

\footnotetext{
Reports available from: http://eprints.maths.manchester.ac.uk/

And by contacting: The MIMS Secretary

School of Mathematics

The University of Manchester

Manchester, M13 9PL, UK
} 


\title{
CONSTRUCTING STRONG LINEARIZATIONS OF MATRIX POLYNOMIALS EXPRESSED IN CHEBYSHEV BASES*
}

\author{
PIERS W. LAWRENCE ${ }^{\dagger}$ AND JAVIER PÉREZ
}

\begin{abstract}
The need to solve polynomial eigenvalue problems for matrix polynomials expressed in nonmonomial bases has become a very important problem. Among the most important bases in numerical applications are the Chebyshev polynomials of the first and second kind. In this work, we introduce a new approach for constructing strong linearizations for matrix polynomials expressed in Chebyshev bases, generalizing the classical colleague pencil, and expanding the arena in which to look for linearizations of matrix polynomials expressed in Chebyshev bases. We show that any of these linearizations is a strong linearization regardless whether the matrix polynomial is regular or singular. In addition, we show how to recover eigenvectors, minimal indices, and minimal bases of the polynomial from those of any of the new linearizations. As an example, we also construct strong linearizations for matrix polynomials of odd degree that are symmetric whenever the matrix polynomials are symmetric.
\end{abstract}

Key words. Chebyshev polynomials, Chebyshev pencils, strong linearizations, matrix polynomials, singular matrix polynomials, eigenvector recovery, minimal bases, minimal indices, one-sided factorizations, structure-preserving linearizations

AMS subject classifications. 15A22, 15A18, 15A23, 65H04, 65F 15

1. Introduction. Chebyshev polynomials are widely used in many areas of numerical analysis, especially in approximation theory $[12,32,26,31]$. A common approach to solving the nonlinear eigenvalue problem

$$
T(\lambda) x=0, \quad y^{*} T(\lambda)=0
$$

for a holomorphic matrix-valued function $T: \Omega \rightarrow \mathbb{C}^{n \times n}$, is to construct a matrix polynomial approximation $P(\lambda)$ of the function $T(\lambda)$, replacing the nonlinear eigenvalue problem with the polynomial eigenvalue problem

$$
P(\lambda) x=0, \quad y^{*} P(\lambda)=0
$$

via Chebyshev interpolation [12].

One of the most common approaches to solve polynomial eigenvalue problems is to linearize the matrix polynomial. Linearization transforms the polynomial problem into an equivalent generalized eigenvalue problem - that is, a matrix pencil or a linear matrix polynomial - for which standard techniques such as the QZ algorithm can be used to recover the eigenstructure [23].

The linearization transformation is not unique $[7,22]$. In practice, the most frequently used linearization to solve a polynomial eigenvalue problem is the Frobenius companion form, which can be constructed directly from the coefficients of the matrix

*The first author has been partially supported by the Belgian Network DYSCO (Dynamical Systems, Control, and Optimization), funded by the Interuniversity Attraction Poles Programme initiated by the Belgian Science Policy Office, and the second author has been supported by Engineering and Physical Sciences Research Council grant EP/I005293.

†Université catholique de Louvain, Department of Mathematical Engineering, Avenue Georges Lemaître 4, B-1348 Louvain-la-Neuve, Belgium.

${ }^{\dagger}$ KU Leuven, Dept. Computer Science, Celestijnenlaan 200A bus 2402, B-3001 Leuven, Belgium. (piers.lawrence@cs.kuleuven.be).

$\ddagger$ School of Mathematics, The University of Manchester, Manchester, England, M13 9PL. (javier.perezalvaro@manchester.ac.uk) 
polynomial when the polynomial is expressed in the monomial basis [7]. However, it is also very desirable to have the ability to construct large families of linearizations from which one can select the linearization that has the most favorable properties, such as conditioning [17], backward errors [10, 16, 19, 27, 30], or the preservation of structure $[6,8,22,21]$, for example.

On the other hand, when solving polynomial eigenvalue problems expressed in a nonmonomial basis, it is important to avoid reformulating the matrix polynomial into the monomial one $[12,31]$, since this change of basis can be poorly conditioned, and may introduce numerical errors. For this reason, constructing linearizations of matrix polynomials from the (nonmonomial) coefficients of $P(\lambda)$ in a nonmonomial basis has become an important need $[24,25,28,29]$. In this work, we consider the problem of constructing (strong) linearizations of matrix polynomials expressed in the first- or second-kind Chebyshev polynomial bases.

The most well-known linearization easily built from the coefficients of a square matrix polynomial expressed in the Chebyshev basis is the colleague pencil (3.3) introduced by Good [15]. Recently, a family of linearizations for square matrix polynomials expressed in the Chebyshev basis was introduced in [29]. This family was called Fiedler-Chebyshev pencils, and it contains the colleague pencil (3.3) as a particular case. Unfortunately, the same approach in [29] cannot be easily extended to rectangular matrix polynomials. Furthermore, none of the linearizations belonging to the Fiedler-Chebyshev pencil family reflect the same structure that the matrix polynomial might possess [21].

In this work, we propose a different approach to the one taken in [29]. By adapting the approach in [11] to tackle Chebyshev polynomials, we provide a rich source of new linearizations for matrix polynomials expressed in Chebyshev bases. We refer to any of these linearizations as Chebyshev linearizations, and we prove that they are strong linearizations, regardless of whether the matrix polynomial is regular, singular, square or rectangular. Moreover, to illustrate the power and flexibility of this approach, we will construct through a number of examples several block symmetric strong linearizations of matrix polynomials with odd degree.

Following this introduction, the rest of the paper is structured into three additional sections. Section 2 outlines the the notation and reviews the basic definitions and previous results used in the paper. In Section 3, we introduce a class of block anti-triangular pencils (which we call Chebyshev linearizations), and we outline a simple procedure to easily generate strong linearizations of matrix polynomials expressed in Chebyshev bases from Chebyshev pencils. Finally, Section 4 demonstrates how the eigenvectors, minimal bases, and minimal indices of a matrix polynomial and those of any Chebyshev pencil are related, thus allowing us to give simple recipes for recovering them.

2. Basic results and notation. In this section, we recall some definitions and results relating to matrix polynomials. Throughout the paper we use the following notation: given an arbitrary field $\mathbb{F}$, we denote by $\mathbb{F}[\lambda]$ the ring of all univariate polynomials in the variable $\lambda$ with coefficients in $\mathbb{F}$, and by $\mathbb{F}(\lambda)$ we denote the field of rational functions with coefficients in $\mathbb{F}$. By $\mathbb{F}[\lambda]^{m \times n}$ and $\mathbb{F}(\lambda)^{m \times n}$ we denote, respectively, the set of $m \times n$ matrices with entries in $\mathbb{F}[\lambda]$ and the set of $m \times n$ matrices with entries in $\mathbb{F}(\lambda)$.

A matrix $P(\lambda) \in \mathbb{F}[\lambda]^{m \times n}$ is called a $m \times n$ matrix polynomial. In this work, we 
will focus on matrix polynomials of the form

$$
P(\lambda)=\sum_{k=0}^{d} P_{k} \phi_{k}(\lambda) \in \mathbb{F}[\lambda]^{m \times n},
$$

where all of the polynomials $\phi_{0}(\lambda), \ldots, \phi_{d}(\lambda)$ are either Chebyshev polynomials of the first kind or of the the second kind. Chebyshev polynomials of the first kind, denoted by $\left\{T_{i}(\lambda)\right\}$, satisfy the recurrence relation $T_{0}(\lambda)=1, T_{1}(\lambda)=\lambda$, and

$$
T_{i}(\lambda)=2 \lambda T_{i-1}(\lambda)-T_{i-2}(\lambda) \quad i \geq 2 .
$$

Chebyshev polynomials of the second kind, denoted by $\left\{U_{i}(\lambda)\right\}$, satisfy the recurrence relation $U_{0}(\lambda)=1, U_{1}(\lambda)=2 \lambda$, and

$$
U_{i}(\lambda)=2 \lambda U_{i-1}(\lambda)-U_{i-2}(\lambda) \quad i \geq 2 .
$$

If the leading matrix coefficient $P_{d}$ in (2.1) is not equal to the $m \times n$ zero matrix, the quantity $d$ is called the degree of $P(\lambda)$; otherwise, $d$ is called the grade of $P(\lambda)$ (also known as its extended degree [18]). We recall that a matrix polynomial of degree $d$ can be considered as a matrix polynomial of any grade larger than or equal to $d$. Throughout this paper when the grade is not explicitly stated, we consider its grade as its degree. We will denote the degree of a matrix polynomial $P(\lambda)$ by $\operatorname{deg}(P(\lambda)$ ). A matrix polynomial of grade 1 is called a matrix pencil.

For any $k \geq \operatorname{deg}(P(\lambda))$ the $k$-reversal matrix polynomial of $P(\lambda)$ is

$$
\operatorname{rev}_{k} P(\lambda):=\lambda^{k} P\left(\lambda^{-1}\right) .
$$

If the degree of the polynomial $P(\lambda)$ is clear, then we will sometimes write $\operatorname{rev} P(\lambda)$ instead of $\operatorname{rev}_{k} P(\lambda)$, where $k=\operatorname{deg}(P(\lambda))$. Notice that $\operatorname{rev}_{k} P(\lambda)$ is also a matrix polynomial.

A matrix polynomial $P(\lambda)$ is said to be regular if $P(\lambda)$ is square and $\operatorname{det} P(\lambda)$ is not identically zero. Otherwise, the matrix polynomial $P(\lambda)$ is said to be singular. The rank of $P(\lambda)$, denoted $\operatorname{rank} P(\lambda)$, is the rank of $P(\lambda)$ when viewed as a matrix with entries in the field $\mathbb{F}(\lambda)$. For the eigenstructure of a matrix polynomial we will follow the same notation and definitions as in [9, Definition 2.17]. We recall that the complete eigenstructure of a matrix polynomial consists of its finite and infinite elementary divisors, together with its left and right minimal indices.

Singular matrix polynomials may have non-empty right and left nullspaces. These vector spaces are introduced in the following definition; henceforth we refer to any matrix polynomial $P(\lambda) \in \mathbb{F}[\lambda]^{m \times 1}$ as a vector polynomial.

DEFINITION 2.1. The left and right nullspaces of a singular matrix polynomial $P(\lambda) \in \mathbb{F}[\lambda]^{m \times n}$, denoted by $\mathcal{N}_{l}(P)$ and $\mathcal{N}_{r}(P)$, respectively, are the vector spaces

$$
\begin{aligned}
& \mathcal{N}_{l}(P):=\left\{y(\lambda)^{T} \in \mathbb{F}(\lambda)^{1 \times m} \quad \text { such that } \quad y(\lambda)^{T} P(\lambda)=0\right\}, \\
& \mathcal{N}_{r}(P):=\left\{x(\lambda) \in \mathbb{F}(\lambda)^{n \times 1} \quad \text { such that } P(\lambda) x(\lambda)=0\right\} .
\end{aligned}
$$

It is always possible to find bases for $\mathcal{N}_{l}(P)$ and $\mathcal{N}_{r}(P)$ consisting entirely of vector polynomials. Indeed, given any basis $\left\{x_{1}(\lambda), \ldots, x_{r}(\lambda)\right\}$, multiply every vector $x_{j}(\lambda)$ by the least common denominator of all entries of $x_{1}(\lambda), \ldots, x_{r}(\lambda)$. The order of a vector polynomial basis is defined as the sum of the degrees of its vectors [14, 
Definition 2]. Among all the possible polynomial bases of $\mathcal{N}_{l}(P)$ and $\mathcal{N}_{r}(P)$, we are interested in the ones with least order.

Definition 2.2. [14, Definition 3] Let $\mathcal{V}$ be a subspace of $\mathbb{F}(\lambda)^{n \times 1}$. A minimal basis of $\mathcal{V}$ is any polynomial basis of $\mathcal{V}$ with least order among all polynomial bases.

The minimal bases of $\mathcal{N}_{l}(P)$ and $\mathcal{N}_{r}(P)$ are not unique, but the ordered list of degrees of the vector polynomials in any minimal basis of $\mathcal{V}$ is always the same. This motivates our next definition (see [14, Definition 4]).

Definition 2.3. Let $P(\lambda) \in \mathbb{F}[\lambda]^{m \times n}$ be a singular matrix polynomial, and let $\left\{y_{1}(\lambda)^{T}, \ldots, y_{q}(\lambda)^{T}\right\}$ and $\left\{x_{1}(\lambda), \ldots, x_{p}(\lambda)\right\}$ be minimal bases of $\mathcal{N}_{l}(P)$ and $\mathcal{N}_{r}(P)$, respectively, ordered such that $\operatorname{deg}\left(y_{1}(\lambda)\right) \leq \cdots \leq \operatorname{deg}\left(y_{q}(\lambda)\right)$ and $\operatorname{deg}\left(x_{1}(\lambda)\right) \leq \cdots \leq$ $\operatorname{deg}\left(x_{p}(\lambda)\right)$. Let $\mu_{j}=\operatorname{deg}\left(y_{j}(\lambda)\right)$, for $j=1,2, \ldots, q$, and $\epsilon_{j}=\operatorname{deg}\left(x_{j}(\lambda)\right)$, for $j=$ $1,2, \ldots, p$. Then, $\mu_{1} \leq \cdots \leq \mu_{q}$ and $\epsilon_{1} \leq \cdots \leq \epsilon_{p}$ are, respectively, the left and right minimal indices of $P(\lambda)$.

Finally, a linearization of a matrix polynomial $P(\lambda)$ of grade $d$ is a pencil $L(\lambda)=$ $\lambda B+A$ such that there exist two unimodular matrices (i.e., having constant nonzero determinant) $U(\lambda)$ and $V(\lambda)$ satisfying

$$
U(\lambda) L(\lambda) V(\lambda)=\left[\begin{array}{ll}
I_{s} & \\
& P(\lambda)
\end{array}\right],
$$

for some natural number $s$, and where $I_{s}$ denotes the $s \times s$ identity matrix [9]. In addition, the pencil $L(\lambda)$ is said to be a strong linearization if $\operatorname{rev}_{1} L(\lambda)=\lambda A+B$ is a linearization of $\operatorname{rev}_{d} P(\lambda)$, that is, if there exist unimodular matrix polynomials $\widehat{U}(\lambda)$ and $\widehat{V}(\lambda)$ such that

$$
\widehat{U}(\lambda) \operatorname{rev}_{1} L(\lambda) \widehat{V}(\lambda)=\left[\begin{array}{ll}
I_{s} & \\
& \operatorname{rev}_{d} P(\lambda)
\end{array}\right] .
$$

We recall that the key property of any strong linearization $L(\lambda)$ of $P(\lambda)$ is that $L(\lambda)$ preserves the finite and infinite eigenstructure of $P(\lambda)$ as well as the dimensions of the right and left null spaces of $P(\lambda)$ (see, for example, [9]). On the other hand, it is well known that linearizations may change right and left minimal indices arbitrarily [9, Theorem 4.11].

3. Constructing strong linearizations of matrix polynomials expressed in Chebyshev bases. The aim of this section is to construct strong linearizations of the matrix polynomial (2.1) that are constructible directly from its matrix coefficients. To do so, we first introduce the concept of a Chebyshev pencil.

3.1. Chebyshev pencils. We will refer to any pencil with the following block anti-triangular structure as a Chebyshev pencil:

$$
\begin{aligned}
& \mathcal{L}(\lambda)=\left[\begin{array}{c|c}
\lambda M_{1}+M_{0} & C_{\mu}^{(j)}(\lambda)^{T} \otimes I_{m} \\
\hline C_{\epsilon}^{(i)}(\lambda) \otimes I_{n} & 0
\end{array}\right] \quad\left\{\begin{array}{l}
(\mu+1) m \\
\epsilon n,
\end{array}\right. \\
& \underbrace{}_{(\epsilon+1) n} \underbrace{}_{\mu m}
\end{aligned}
$$


where $i, j \in\{1,2\}$,

$$
C_{k}^{(j)}(\lambda)=\left[\begin{array}{cccccc}
1 & -2 \lambda & 1 & & & \\
& 1 & -2 \lambda & 1 & & \\
& & \ddots & \ddots & \ddots & \\
& & & 1 & -2 \lambda & 1 \\
& & & & 1 & -j \lambda
\end{array}\right] \in \mathbb{F}[\lambda]^{k \times(k+1)}
$$

and where $\otimes$ denotes the Kronecker product.

REMARK 1. Let us consider a square $(n=m)$ matrix polynomial $P(\lambda)$ expressed in one of the Chebyshev bases (either the first or the second kind), as in (2.1). Then, it is well known that the pencil

$$
\lambda\left[\begin{array}{ccccc}
2 P_{d} & & & & \\
& -2 I_{n} & & & \\
& & \ddots & & \\
& & & -2 I_{n} & \\
& & & & -j I_{n}
\end{array}\right]+\left[\begin{array}{ccccc}
P_{d-1} & P_{d-2}-P_{d} & P_{d-3} & \cdots & P_{0} \\
I_{n} & 0 & I_{n} & & \\
& \ddots & \ddots & \ddots & \\
& & I_{n} & 0 & I_{n} \\
& & & I_{n} & 0
\end{array}\right],
$$

is a strong linearization for $P(\lambda)$, where $j=1,2$ correspond to Chebyshev polynomials of the first and second kind [2], respectively. This pencil is a particular case of a comrade pencil [3], which is a linearization of a matrix polynomial expressed in an orthogonal polynomial basis (for $j=1$ or $j=2$ it is also called a colleague pencil). Notice that the pencil (3.3) is a Chebyshev pencil with

$$
\lambda M_{1}+M_{0}=\left[\begin{array}{llllll}
2 \lambda P_{d}+P_{d-1} & P_{d-2}-P_{d} & P_{d-3} & \cdots & P_{1} & P_{0}
\end{array}\right],
$$

and $\epsilon=d-1$ and $\mu=0$.

In Section 3.3 we prove that the Chebyshev pencil (3.1) is always a strong linearization of a certain matrix polynomial. This result allows us to construct strong linearizations for the matrix polynomial, as we show in in Section 3.4. The tools and auxiliary results needed to construct the unimodular matrix polynomials in the linearization transformations (2.4) and (2.5) are introduced in the following section.

3.2. Auxiliary unimodular matrix polynomials. Here we will identify the appropriate unimodular matrix polynomials in (2.4) and (2.5) for the Chebyshev pencil (3.1).

It is clear that the pencils $C_{k}^{(j)}(\lambda)$ in (3.2) have full row rank for any $\lambda$. A key property of any matrix polynomial $Q(\lambda)$ with this property is that it can be embedded in a unimodular matrix polynomial

$$
\widehat{Q}(\lambda)=\left[\begin{array}{l}
Q(\lambda) \\
\widetilde{Q}(\lambda)
\end{array}\right]
$$

such that $\operatorname{deg}(\widetilde{Q}(\lambda)) \leq \operatorname{deg}(Q(\lambda))-1$ (see, for example, [5, Corollary 1]). Specializing this result to a matrix polynomial $Q(\lambda)$ of degree 1 and size $k \times(k+1), Q(\lambda)$ may be embedded in a unimodular matrix pencil $\widehat{Q}(\lambda)$, where $\widetilde{Q}(\lambda)$ is just a constant row 
vector. For the matrix pencil $C_{k}^{(j)}(\lambda)$ in (3.2), this result is evident, since

$$
V_{k}^{(j)}(\lambda):=\left[\frac{C_{k}^{(j)}(\lambda)}{e_{k+1}}\right]=\left[\begin{array}{cccccc}
1 & -2 \lambda & 1 & & & \\
& 1 & -2 \lambda & 1 & & \\
& & \ddots & \ddots & \ddots & \\
& & & 1 & -2 \lambda & 1 \\
& & & & 1 & -j \lambda \\
\hline & & & & & 1
\end{array}\right] \in \mathbb{F}[\lambda]^{(k+1) \times(k+1)}
$$

is clearly unimodular. Moreover, a unimodular matrix polynomial has a polynomial inverse, and for the pencil $V_{k}^{(j)}(\lambda)$ in (3.4), an explicit expression is easily derived, as we show in the following lemma.

Lemma 3.1. Let $V_{k}^{(j)}(\lambda)$ be the pencil in (3.4) with $j \in\{1,2\}$. Then, its inverse is given by

$$
V_{k}^{(j)}(\lambda)^{-1}=\left[\begin{array}{ll}
M_{k}(\lambda) & \Pi_{k}^{(j)}(\lambda)
\end{array}\right]
$$

where

$$
M_{k}(\lambda)=\left[\begin{array}{cccc}
U_{0}(\lambda) & U_{1}(\lambda) & \ldots & U_{k-1}(\lambda) \\
& \ddots & \ddots & \vdots \\
& & U_{0}(\lambda) & U_{1}(\lambda) \\
& & & U_{0}(\lambda) \\
0 & \cdots & 0 & 0
\end{array}\right] \in \mathbb{F}[\lambda]^{(k+1) \times k}
$$

and where

$$
\Pi_{k}^{(1)}(\lambda)=\left[\begin{array}{c}
T_{k}(\lambda) \\
\vdots \\
T_{1}(\lambda) \\
T_{0}(\lambda)
\end{array}\right] \in \mathbb{F}[\lambda]^{(k+1) \times 1}, \text { and } \Pi_{k}^{(2)}(\lambda)=\left[\begin{array}{c}
U_{k}(\lambda) \\
\vdots \\
U_{1}(\lambda) \\
U_{0}(\lambda)
\end{array}\right] \in \mathbb{F}[\lambda]^{(k+1) \times 1} \text {. }
$$

Proof. Using the recurrence relations (2.2) and (2.3), it is immediately verified that $V_{k}^{(j)}(\lambda)\left[\begin{array}{ll}M_{k}(\lambda) & \Pi_{k}^{(j)}(\lambda)\end{array}\right]=I_{k+1}$.

The reversal of the pencil (3.2) is given by

$$
\operatorname{rev}_{1} C_{k}^{(j)}(\lambda)=\left[\begin{array}{cccccc}
\lambda & -2 & \lambda & & & \\
& \lambda & -2 & \lambda & & \\
& & \ddots & \ddots & \ddots & \\
& & & \lambda & -2 & \lambda \\
& & & & \lambda & -j
\end{array}\right] \in \mathbb{E}[\lambda]^{k \times(k+1)} .
$$

Notice that the pencil (3.8) also has full row rank for any $\lambda$. This implies that it can also be embedded in a unimodular matrix by appending a constant row vector

$$
W_{k}^{(j)}(\lambda):=\left[\frac{\left(\omega^{(j)}\right)^{T}}{\operatorname{rev}_{1} C_{k}^{(j)}(\lambda)}\right]=\left[\begin{array}{cccccc}
\omega_{k}^{(j)} & \omega_{k-1}^{(j)} & \omega_{k-2}^{(j)} & \cdots & \omega_{1}^{(j)} & \omega_{0}^{(j)} \\
\hline \lambda & -2 & \lambda & & & \\
& \lambda & -2 & \lambda & & \\
& & \ddots & \ddots & \ddots & \\
& & & \lambda & -2 & \lambda \\
& & & & \lambda & -j
\end{array}\right],
$$


where, for convenience, the vector is appended in the first row. The vector $\omega^{(j)}$ in (3.9) must now be chosen to guarantee that $W_{k}^{(j)}(\lambda)$ is unimodular, one such choice is to ensure that $\operatorname{det} W_{k}^{(j)}(\lambda)=1$, as we will now show.

Lemma 3.2. Let $W_{k}^{(j)}(\lambda)$ be the pencil in (3.9) with $j \in\{1,2\}$. If we set

$$
\omega_{i}^{(1)}:= \begin{cases}(-1)^{k} 2^{1-k}\left(\begin{array}{c}
k \\
(k-i) / 2
\end{array}\right) & \text { if } k-i \text { is even and } i \neq 0, \\
2^{-k}\left(\begin{array}{c}
k \\
k / 2
\end{array}\right) & \text { if } k \text { is even and } i=0, \quad \text { and } \\
0 & \text { otherwise, }\end{cases}
$$

and

$\omega_{i}^{(2)}:= \begin{cases}(-1)^{k} 2^{-k}\left(\left(\begin{array}{c}k \\ (k-i) / 2\end{array}\right)-\left(\begin{array}{c}k \\ (k-i-2) / 2\end{array}\right)\right) & \text { if } k-i \text { is even and } i \neq 0, \\ 0 & \text { otherwise }\end{cases}$

for $i=0,1, \ldots, k$, then $\operatorname{det} W_{k}^{(j)}(\lambda)=1$, for $j=1,2$.

Proof. We first use the properties of the $k$-reversal operation in order to obtain an expression for the determinant $\operatorname{det} W_{k}^{(j)}(\lambda)$. The following sequence of equalities holds:

$$
\operatorname{det} W_{k}^{(j)}(\lambda)=\operatorname{det}\left(\operatorname{rev}_{1}\left(\operatorname{rev}_{1} W_{k}^{(j)}(\lambda)\right)\right)=\operatorname{rev}_{k+1}\left(\operatorname{det}\left(\operatorname{rev}_{1} W_{k}^{(j)}(\lambda)\right)\right),
$$

and the determinant of interest is now

$$
\operatorname{det}\left(\operatorname{rev}_{1} W_{k}^{(j)}(\lambda)\right)=\operatorname{det}\left[\begin{array}{ccccc}
\lambda \omega_{k}^{(j)} & \lambda \omega_{k-1}^{(j)} & \cdots & \lambda \omega_{1}^{(j)} & \lambda \omega_{0}^{(j)} \\
\hline 1 & -2 \lambda & 1 & & \\
& \ddots & \ddots & \ddots & \\
& & 1 & -2 \lambda & 1 \\
& & & 1 & -j \lambda
\end{array}\right] .
$$

The pencil above can be shown to be a linearization of the polynomial

$$
q^{(j)}(\lambda)=(-1)^{k} \lambda \sum_{i=0}^{k} \omega_{i}^{(j)} \phi_{i}^{(j)}(\lambda)
$$

where the $\phi_{k}^{(1)}(\lambda)=T_{k}(\lambda)$, and $\phi_{k}^{(2)}(\lambda)=U_{0}(\lambda)$. This may be established using the linearization in $[19,(3.2)]$, or via Laplace expansion along the first column of the pencil in (3.11). Such an expansion yields

$$
\operatorname{det}\left(\operatorname{rev}_{1} W_{k}^{(j)}(\lambda)\right)=\sum_{i=0}^{k} \lambda \omega_{i}^{(j)}(-1)^{k-i} \operatorname{det}\left(S_{i}-\lambda T_{i}^{(j)}\right)=q^{(j)}(\lambda),
$$

where we have used the well-known determinants [15] for $j \in\{1,2\}$ to obtain

$$
\operatorname{det}\left(S_{i}-\lambda T_{i}^{(j)}\right)=\operatorname{det} \underbrace{\left[\begin{array}{ccccc}
-2 \lambda & 1 & & & \\
1 & -2 \lambda & 1 & & \\
& \ddots & \ddots & \ddots & \\
& & 1 & -2 \lambda & 1 \\
& & & 1 & -j \lambda
\end{array}\right]}_{i}=(-1)^{i} \phi_{i}^{(j)}(\lambda) .
$$


Finally, substituting (3.12) into (3.10) yields

$$
\operatorname{det} W_{k}^{(j)}=(-1)^{k} \sum_{i=0}^{k} \omega_{i}^{(j)} \lambda^{k} \phi_{i}^{(j)}(1 / \lambda)=(-1)^{k} \operatorname{rev}_{k} \sum_{i=0}^{k} \omega_{i}^{(j)} \phi_{i}^{(j)}(\lambda),
$$

which we equate to 1 and take the $k$-reversal to obtain the entries $\omega_{i}^{(j)}$. These are exactly the coefficients of the expansion of $(-\lambda)^{k}$ in a Chebyshev basis, that is, satisfying

$$
(-\lambda)^{k}=\sum_{i=0}^{k} \omega_{i}^{(j)} \phi_{i}^{(j)}(\lambda)
$$

This expansion is a known result [1]. For $j=1$, it is given by

$$
(-\lambda)^{k}=(-1)^{k} 2^{1-k} \sum_{\substack{0 \leq i \leq k \\
k-i \text { even }}}^{\prime}\left(\begin{array}{c}
k \\
(k-i) / 2
\end{array}\right) T_{i}(\lambda),
$$

where the primed sum indicates that the contribution of $i=0$ needs to be halved if it appears. This expansion, together with the formula $T_{i}(\lambda)=\left(U_{i}(\lambda)-U_{i-2}(\lambda)\right) / 2$ implies the desired result that

$$
(-\lambda)^{k}=(-1)^{k} 2^{-k} \sum_{\substack{0 \leq i \leq k \\
k-i \text { even }}}\left(\left(\begin{array}{c}
k \\
(k-i) / 2
\end{array}\right)-\left(\begin{array}{c}
k \\
(k-i-2) / 2
\end{array}\right)\right) U_{i}(\lambda) .
$$

In contrast to the result in Lemma 3.1, an explicit formula for $W_{k}^{(j)}(\lambda)^{-1}$ is not as simple to obtain. However, since $W_{k}^{(j)}(\lambda)$ is unimodular, it has a polynomial inverse, and thus we can compute its expansion in a Chebyshev basis using Barnett's [4] extension of the Leverrier-Faddeev [13, 20] algorithm. For our purposes here we only require the first column of the inverse $W_{k}^{(j)}(\lambda)^{-1}$ in order to establish the strong linearization property, we thus offer the following lemma.

Lemma 3.3. For $j \in\{1,2\}$, let $W_{k}^{(j)}(\lambda)$ be the pencil in (3.9) with the vector $\omega^{(j)}$ as in Lemma 3.2. The inverse of $W_{k}^{(j)}(\lambda)$ is of the form

$$
W_{k}^{(j)}(\lambda)^{-1}=\left[\operatorname{rev}_{k} \Pi_{k}^{(j)}(\lambda) \quad N_{k}^{(j)}(\lambda)\right],
$$

where $\Pi_{k}^{(j)}(\lambda)$ is defined in (3.7), and where $N_{k}^{(j)}(\lambda)$ is a matrix polynomial such that $\operatorname{deg}\left(N_{k}^{(j)}(\lambda)\right) \leq k-1$.

Proof. Let us introduce the notation

$$
W_{k}^{(j)}(\lambda)^{-1}=\left[\begin{array}{ll}
f(\lambda) & N_{k}^{(j)}(\lambda)
\end{array}\right]
$$

where $f(\lambda)$ corresponds to the first column of $W_{k}^{(j)}(\lambda)^{-1}$. From

$$
W_{k}^{(j)}(\lambda) W_{k}^{(j)}(\lambda)^{-1}=\left[\begin{array}{c}
\left(\omega^{(j)}\right)^{T} \\
\operatorname{rev}_{1} C_{k}^{(j)}(\lambda)
\end{array}\right]\left[\begin{array}{ll}
f(\lambda) & N_{k}^{(j)}(\lambda)
\end{array}\right]=\left[\begin{array}{cc}
1 & 0 \\
0 & I_{k}
\end{array}\right],
$$


we see that $f(\lambda)$ must belong to the null space of $\operatorname{rev}_{1} C_{k}^{(j)}(\lambda)$, which is a vector space of dimension 1 (note that $\operatorname{rev}_{1} C_{k}^{(j)}(\lambda)$ has full row rank and size $k \times(k+1)$ ). Direct matrix multiplication allows us to immediately verify that the vector $\operatorname{rev}_{k} \Pi_{k}^{(j)}(\lambda)$ belongs to the null space of $\operatorname{rev}_{1} C_{k}^{(j)}(\lambda)$. It follows that $f(\lambda)=\alpha(\lambda) \operatorname{rev}_{k} \Pi_{k}^{(j)}(\lambda)$ for some polynomial function $\alpha(\lambda)$. Inspecting the $(1,1)$ entry, we additionally have $1=\left(\omega^{(j)}\right)^{T} f(\lambda)=\alpha(\lambda)\left(\omega^{(j)}\right)^{T} \operatorname{rev}_{k} \Pi_{k}^{(j)}(\lambda)=\alpha(\lambda)$, and thus $f(\lambda)=\operatorname{rev}_{k} \Pi_{k}^{(j)}(\lambda)$.

Finally, that the degree of the matrix polynomial $N_{k}^{(j)}(\lambda)$ is bounded by $k-1$ follows from the following argument. First, notice that

$$
W_{k}^{(j)}(\lambda)^{-1}=\operatorname{adj}\left(W_{k}^{(j)}(\lambda)\right) / \operatorname{det}\left(W_{k}^{(j)}(\lambda)\right)=\operatorname{adj}\left(W_{k}^{(j)}(\lambda)\right),
$$

where adj $\left(W_{k}^{(j)}(\lambda)\right)$ is the classical adjoint of $W_{k}^{(j)}(\lambda)$. Then, to compute any of the entries of the last $k$ columns of adj $\left(W_{k}^{(j)}(\lambda)\right)$ via cofactors, one has to compute the determinant of a $k \times k$ matrix. The first row of this matrix is constant while the entries of the other $k-1$ rows are polynomials with degree at most 1 . Therefore, the degree of the entries of the last $k-1$ rows of adj $\left(W_{k}^{(j)}(\lambda)\right)$ are bounded by $k-1$.

3.3. Chebyshev pencils as strong linearizations. We are now in a position to prove Theorem 3.4, where we show that the Chebyshev pencil (3.1) is a strong linearization of the matrix polynomial (3.18).

TheOREm 3.4. Let $\mathcal{L}(\lambda)$ be a Chebyshev pencil as in (3.1). Then, the pencil $\mathcal{L}(\lambda)$ is a strong linearization of the matrix polynomial

$$
P(\lambda)=\left(\Pi_{\mu}^{(j)}(\lambda)^{T} \otimes I_{m}\right)\left(\lambda M_{1}+M_{0}\right)\left(\Pi_{\epsilon}^{(i)}(\lambda) \otimes I_{n}\right),
$$

of grade $d:=\epsilon+\mu+1$.

Proof. We first show that $\mathcal{L}(\lambda)$ is a linearization of $P(\lambda)$, that is, we construct unimodular matrix polynomials $U(\lambda)$ and $V(\lambda)$ such that (2.4) holds. We make use of the unimodular matrix polynomial $V_{k}^{(j)}(\lambda)^{-1}$, with $j \in\{1,2\}$, introduced in (3.5), embedding it in the following unimodular matrices:

$$
U_{1}(\lambda):=\left[\begin{array}{ll}
V_{\mu}^{(j)}(\lambda)^{-T} \otimes I_{m} & \\
& I_{\epsilon n}
\end{array}\right]=\left[\begin{array}{ccc}
M_{\mu}(\lambda)^{T} \otimes I_{m} & \\
\Pi_{\mu}^{(j)}(\lambda)^{T} \otimes I_{m} & \\
& & I_{\epsilon n}
\end{array}\right],
$$

and

$$
V_{1}(\lambda):=\left[\begin{array}{ll}
V_{\epsilon}^{(i)}(\lambda)^{-1} \otimes I_{n} & \\
& I_{\mu m}
\end{array}\right]=\left[\begin{array}{lll}
M_{\epsilon}(\lambda) \otimes I_{n} & \Pi_{\epsilon}^{(i)}(\lambda) \otimes I_{n} & \\
& & I_{\mu m}
\end{array}\right] .
$$

Pre- and post-multiplying the Chebyshev pencil by $U_{1}(\lambda)$ and $V_{1}(\lambda)$, respectively, we obtain

$$
\left[\begin{array}{ccc}
Z(\lambda) & X(\lambda) & I_{\mu m} \\
Y(\lambda) & P(\lambda) & 0 \\
I_{\epsilon n} & 0 & 0
\end{array}\right]
$$

where the matrix polynomials $X(\lambda), Y(\lambda)$ and $Z(\lambda)$ are given by

$$
\begin{aligned}
& X(\lambda)=\left(M_{\mu}(\lambda)^{T} \otimes I_{m}\right)\left(\lambda M_{1}+M_{0}\right)\left(\Pi_{\epsilon}^{(i)}(\lambda) \otimes I_{n}\right), \\
& Y(\lambda)=\left(\Pi_{\mu}^{(j)}(\lambda)^{T} \otimes I_{m}\right)\left(\lambda M_{1}+M_{0}\right)\left(M_{\epsilon}(\lambda) \otimes I_{n}\right), \quad \text { and } \\
& Z(\lambda)=\left(M_{\mu}(\lambda)^{T} \otimes I_{m}\right)\left(\lambda M_{1}+M_{0}\right)\left(M_{\epsilon}(\lambda) \otimes I_{n}\right) .
\end{aligned}
$$


Because we have reduced the anti-diagonal blocks to identity matrices, it is clear that these may be used to annihilate the entries $Z(\lambda), Y(\lambda)$, and $X(\lambda)$ in the above matrix. Explicitly, we obtain the following transformation:

$$
\begin{aligned}
& {\left[\begin{array}{ccc}
I_{\mu m} & 0 & -Z(\lambda) \\
0 & 0 & I_{\epsilon n} \\
0 & I_{m} & -Y(\lambda)
\end{array}\right]\left[\begin{array}{ccc}
Z(\lambda) & X(\lambda) & I_{\mu m} \\
Y(\lambda) & P(\lambda) & 0 \\
I_{\epsilon n} & 0 & 0
\end{array}\right]\left[\begin{array}{ccc}
0 & I_{\epsilon n} & 0 \\
0 & 0 & I_{n} \\
I_{\mu m} & 0 & -X(\lambda)
\end{array}\right]=} \\
& {\left[\begin{array}{ccc}
I_{\mu m} & & \\
& I_{\epsilon n} & \\
& & P(\lambda)
\end{array}\right],}
\end{aligned}
$$

and thus, the Chebyshev pencil $\mathcal{L}(\lambda)$ is a linearization of $P(\lambda)$.

To show that $\mathcal{L}(\lambda)$ is a strong linearization, we need to prove that $\operatorname{rev}_{1} \mathcal{L}(\lambda)$ is a linearization of $\operatorname{rev}_{d} P(\lambda)$. Thus, we construct unimodular matrices $\widehat{U}(\lambda)$ and $\widehat{V}(\lambda)$ such that (2.5) holds. In this case, we make use of the unimodular matrix polynomial $W_{k}^{(j)}(\lambda)^{-1}$, with $j=1,2$, defined in (3.17). It follows from (3.18) and the properties of the $k$-reversal polynomial that

$$
\operatorname{rev}_{d} P(\lambda)=\left(\operatorname{rev}_{\mu} \Pi_{\mu}^{(j)}(\lambda)^{T} \otimes I_{m}\right)\left(\lambda M_{0}+M_{1}\right)\left(\operatorname{rev}_{\epsilon} \Pi_{\epsilon}^{(i)}(\lambda) \otimes I_{n}\right) .
$$

Let us now introduce the following unimodular matrix polynomials

$$
\widehat{U}_{1}(\lambda):=\left[\begin{array}{ll}
W_{\mu}^{(j)}(\lambda)^{-T} \otimes I_{m} & \\
& I_{\epsilon n}
\end{array}\right]=\left[\begin{array}{cc}
\operatorname{rev}_{\mu} \Pi_{\mu}^{(j)}(\lambda)^{T} \otimes I_{m} & \\
N_{\mu}^{(j)}(\lambda)^{T} \otimes I_{m} & \\
& I_{\epsilon n}
\end{array}\right],
$$

and

$$
\widehat{V}_{1}(\lambda):=\left[\begin{array}{lll}
W_{\epsilon}^{(i)}(\lambda)^{-1} \otimes I_{n} & \\
& I_{\mu m}
\end{array}\right]=\left[\begin{array}{lll}
\operatorname{rev}_{\epsilon} \Pi_{\epsilon}^{(i)}(\lambda) \otimes I_{n} & N_{\epsilon}^{(i)}(\lambda) \otimes I_{n} & \\
& & I_{\mu m}
\end{array}\right] .
$$

Pre- and post-multiplying the pencil $\operatorname{rev}_{1} \mathcal{L}(\lambda)$ by $\widehat{U}_{1}(\lambda)$ and $\widehat{V}_{1}(\lambda)$, respectively, we obtain

$$
\left[\begin{array}{ccc}
\operatorname{rev}_{d} P(\lambda) & \widehat{Y}(\lambda) & 0 \\
\widehat{X}(\lambda) & \widehat{Z}(\lambda) & I_{\mu m} \\
0 & I_{\epsilon n} & 0
\end{array}\right]
$$

where the matrix polynomials $\widehat{X}(\lambda), \widehat{Y}(\lambda)$ and $\widehat{Z}(\lambda)$ are

$$
\begin{aligned}
& \widehat{X}(\lambda)=\left(N_{\mu}^{(j)}(\lambda)^{T} \otimes I_{m}\right)\left(\lambda M_{0}+M_{1}\right)\left(\operatorname{rev}_{\epsilon} \Pi_{\epsilon}^{(i)}(\lambda) \otimes I_{n}\right), \\
& \widehat{Y}(\lambda)=\left(\operatorname{rev}_{\mu} \Pi_{\mu}^{(j)}(\lambda)^{T} \otimes I_{m}\right)\left(\lambda M_{0}+M_{1}\right)\left(N_{\epsilon}^{(j)}(\lambda) \otimes I_{n}\right), \quad \text { and } \\
& \widehat{Z}(\lambda)=\left(N_{\mu}^{(j)}(\lambda)^{T} \otimes I_{m}\right)\left(\lambda M_{0}+M_{1}\right)\left(N_{\epsilon}^{(i)}(\lambda) \otimes I_{n}\right) .
\end{aligned}
$$

Finally, this pencil may be reduced further to

$$
\begin{aligned}
& {\left[\begin{array}{ccc}
0 & 0 & I_{\epsilon n} \\
0 & I_{\mu m} & -\widehat{Z}(\lambda) \\
I_{m} & 0 & -\widehat{Y}(\lambda)
\end{array}\right]\left[\begin{array}{ccc}
\operatorname{rev}_{d} P(\lambda) & \widehat{Y}(\lambda) & 0 \\
\widehat{X}(\lambda) & \widehat{Z}(\lambda) & I_{\mu m} \\
0 & I_{\epsilon n} & 0
\end{array}\right]\left[\begin{array}{ccc}
0 & 0 & I_{n} \\
I_{\epsilon n} & 0 & 0 \\
0 & I_{\mu m} & -\widehat{X}(\lambda)
\end{array}\right]=} \\
& {\left[\begin{array}{ccc}
I_{\epsilon n} & & \\
& I_{\mu m} & \\
& & \operatorname{rev}_{d} P(\lambda)
\end{array}\right],}
\end{aligned}
$$

and thus the pencil $\operatorname{rev}_{1} \mathcal{L}(\lambda)$ is a linearization of $\operatorname{rev}_{d} P(\lambda)$. This establishes that the Chebyshev pencil $\mathcal{L}(\lambda)$ is a strong linearization of the matrix polynomial (3.18). 
3.4. Constructing strong linearizations of a given matrix polynomial expressed in one of the Chebyshev bases. From Theorem 3.4 we obtain the following procedure for constructing strong linearizations of a given matrix polynomial $P(\lambda)$, as in (2.1), from a Chebyshev pencil (3.1):

Step 1 Choose natural numbers $\epsilon$ and $\mu$ such that $\epsilon+\mu=\operatorname{deg}(P(\lambda))-1$.

Step 2 Choose the pencil $\lambda M_{1}+M_{0}$ such that (3.18) is satisfied.

This procedure guarantees that the Chebyshev pencil (3.1) is a strong linearization of $P(\lambda)$. The goal now is to show how to construct the pencil $\lambda M_{1}+M_{0}$ in Step 2 from the coefficients in the Chebyshev basis of $P(\lambda)$. We treat the cases of Chebyshev polynomials of the first and second kind separately.

3.4.1. Chebyshev polynomials of the first kind. We first focus on matrix polynomials of the form

$$
P(\lambda)=P_{d} T_{d}(\lambda)+P_{d-1} T_{d-1}(\lambda)+\cdots+P_{1} T_{1}(\lambda)+P_{0} T_{0}(\lambda) .
$$

In this case, the strong linearizations introduced in this section are based on Chebyshev pencils (3.1) with $i=1$ and $j=2$, or with $i=2$ and $j=1$, where

$$
\mathcal{L}(\lambda)=\left[\begin{array}{c|c}
\lambda M_{1}+M_{0} & C_{\mu}^{(2)}(\lambda)^{T} \otimes I_{m} \\
\hline C_{\epsilon}^{(1)}(\lambda) \otimes I_{n} & 0
\end{array}\right]
$$

We focus on the case $i=1$ and $j=2$ (since the case $i=2$ and $j=1$ is similar).

Let us start by considering the matrices $M_{0}$ and $M_{1}$ in $(3.22)$ as $(\mu+1) \times(\epsilon+1)$ block matrices $\left[M_{0}(i, j)\right]$ and $\left[M_{1}(i, j)\right]$, where $M_{0}(i, j), M_{1}(i, j) \in \mathbb{F}^{m \times n}$. Recall that Theorem 3.4 implies that the Chebyshev pencil (3.22) is a strong linearization of the matrix polynomial (3.21) if $\epsilon+\mu+1=d$ and

$$
\begin{aligned}
P(\lambda)= & \left(\Pi_{\mu}^{(2)}(\lambda)^{T} \otimes I_{m}\right)\left(\lambda M_{1}+M_{0}\right)\left(\Pi_{\epsilon}^{(1)}(\lambda) \otimes I_{n}\right)= \\
& \sum_{r=1}^{\mu+1} \sum_{s=1}^{\epsilon+1}\left(\lambda M_{1}(r, s)+M_{0}(r, s)\right) U_{\mu+1-r}(\lambda) T_{\epsilon+1-s}(\lambda) .
\end{aligned}
$$

The above equation shows the fundamental importance of finding expressions for Chebyshev polynomials of the first kind in terms of products of the form $U_{\ell_{1}}(\lambda) T_{\ell_{2}}(\lambda)$. Lemma 3.5 outlines one way in which this may be done.

LEMma 3.5. Let $\left\{T_{j}(\lambda)\right\}$ and $\left\{U_{j}(\lambda)\right\}$ be, respectively, the Chebyshev polynomials of the first and second kind. Then,

$$
\begin{aligned}
& T_{r+\ell}(\lambda)=U_{r}(\lambda) T_{\ell}(\lambda)-U_{r-1}(\lambda) T_{\ell-1}(\lambda), \quad \text { and } \\
& T_{r+\ell+1}(\lambda)=2 \lambda U_{r}(\lambda) T_{\ell}(\lambda)-U_{r}(\lambda) T_{\ell-1}(\lambda)-U_{r-1}(\lambda) T_{\ell}(\lambda),
\end{aligned}
$$

if $\ell \neq 0$, and where we set $U_{-1}(\lambda):=0$ and $T_{-1}(\lambda):=0$.

Proof. The proof is immediately evident by applying the multiplication formula

$$
U_{r}(\lambda) U_{\ell}(\lambda)=\sum_{k=0}^{\ell} U_{r-\ell+2 k}(\lambda), \quad r \geq \ell,
$$

and the formula $T_{\ell}(\lambda)=\left(U_{\ell}(\lambda)-U_{\ell-2}(\lambda)\right) / 2$. 
Lemma 3.5 presents the key to be able to construct pencils $\lambda M_{1}+M_{0}$ such that (3.23) is satisfied. Before presenting the general procedure to construct $\lambda M_{1}+M_{0}$, we illustrate some specific examples of its application in Example 1, where we show how to choose the pencil $\lambda M_{1}+M_{0}$ in order to linearize a matrix polynomial with a low degree (namely, $d=6$ ).

EXAMPLE 1. Consider the $m \times n$ matrix polynomial $P(\lambda)=\sum_{k=0}^{6} P_{k} T_{k}(\lambda)$, and Chebyshev pencils (3.22) with $\epsilon=3$ and $\mu=2$. Lemma 3.5 allows us to express $P(\lambda)$ as a sum of products of Chebyshev polynomials, that is, as

$$
P(\lambda)=\sum_{i=0}^{6} P_{i} T_{i}(\lambda)=\sum_{i=0}^{\mu=2} \sum_{j=0}^{\epsilon=3}\left(\lambda A_{i j}+B_{i j}\right) U_{i}(\lambda) T_{j}(\lambda) .
$$

One specific choice is

$$
\begin{aligned}
P(\lambda)= & P_{6}\left(2 \lambda U_{2}(\lambda) T_{3}(\lambda)-U_{2}(\lambda) T_{2}(\lambda)-U_{1}(\lambda) T_{3}(\lambda)\right)+ \\
& P_{5}\left(U_{2}(\lambda) T_{3}(\lambda)-U_{1}(\lambda) T_{2}(\lambda)\right)+ \\
& P_{4}\left(2 \lambda U_{2}(\lambda) T_{1}(\lambda)-U_{1}(\lambda) T_{1}(\lambda)-U_{2}(\lambda) T_{0}(\lambda)\right)+ \\
& P_{3}\left(U_{2}(\lambda) T_{1}(\lambda)-U_{1}(\lambda) T_{0}(\lambda)\right)+ \\
& P_{2} U_{0}(\lambda) T_{2}(\lambda)+P_{1} U_{0}(\lambda) T_{1}(\lambda)+P_{0} U_{0}(\lambda) T_{0}(\lambda) .
\end{aligned}
$$

We then set $M_{1}(r, s):=B_{\mu+1-r, \epsilon+1-s}$ and $M_{0}(r, s):=A_{\mu+1-r, \epsilon+1-s}$, for $r=1, \ldots$, $\mu+1$ and $s=1, \ldots, \epsilon+1$, to obtain the pencil

$$
\lambda M_{1}+M_{0}=\left[\begin{array}{cccc}
2 \lambda P_{6}+P_{5} & -P_{6} & 2 \lambda P_{4}+P_{3} & -P_{4} \\
-P_{6} & -P_{5} & -P_{4} & -P_{3} \\
0 & P_{2} & P_{1} & P_{0}
\end{array}\right] .
$$

This guarantees that the Chebyshev pencil

$\left[\begin{array}{cccc|cc}2 \lambda P_{6}+P_{5} & -P_{6} & 2 \lambda P_{4}+P_{3} & -P_{4} & I_{m} & 0 \\ -P_{6} & -P_{5} & -P_{4} & -P_{3} & -2 \lambda I_{m} & I_{m} \\ 0 & P_{2} & P_{1} & P_{0} & -I_{m} & -2 \lambda I_{m} \\ \hline I_{n} & -2 \lambda I_{n} & I_{n} & 0 & 0 & 0 \\ 0 & I_{n} & -2 \lambda I_{n} & I_{n} & 0 & 0 \\ 0 & 0 & I_{n} & -\lambda I_{n} & 0 & 0\end{array}\right]$

is a strong linearization of $P(\lambda)$. Alternatively, we could have also written $P(\lambda)$ as

$$
\begin{aligned}
P(\lambda)= & P_{6}\left(2 \lambda U_{2}(\lambda) T_{3}(\lambda)-U_{2}(\lambda) T_{2}(\lambda)-U_{1}(\lambda) T_{3}(\lambda)\right)+ \\
& P_{5}\left(U_{2}(\lambda) T_{3}(\lambda)-U_{1}(\lambda) T_{2}(\lambda)\right)+ \\
& \frac{1}{4} P_{4}\left(2 \lambda U_{2}(\lambda) T_{1}(\lambda)-U_{1}(\lambda) T_{1}(\lambda)-U_{2}(\lambda) T_{0}(\lambda)\right)+ \\
& \frac{3}{4} P_{4}\left(U_{1}(\lambda) T_{3}(\lambda)-U_{0}(\lambda) T_{2}(\lambda)\right)+ \\
& P_{3} U_{0}(\lambda) T_{3}(\lambda)+P_{2} U_{0}(\lambda) T_{2}(\lambda)+P_{1} U_{0}(\lambda) T_{1}(\lambda)+P_{0} U_{0}(\lambda) T_{0}(\lambda),
\end{aligned}
$$

and for which the Chebyshev pencil

$$
\left[\begin{array}{cccc|cc}
2 \lambda P_{6}+P_{5} & -P_{6} & \frac{1}{2} \lambda P_{4} & -\frac{1}{4} P_{4} & I_{m} & 0 \\
-P_{6}+\frac{3}{4} P_{4} & -P_{5} & -\frac{1}{4} P_{4} & 0 & 2 \lambda I_{m} & -I_{m} \\
P_{3} & P_{2}-\frac{3}{4} P_{4} & P_{1} & P_{0} & -I_{m} & 2 \lambda I_{m} \\
\hline I_{n} & -2 \lambda I_{n} & I_{n} & 0 & 0 & 0 \\
0 & I_{n} & -2 \lambda I_{n} & I_{n} & 0 & 0 \\
0 & 0 & I_{n} & -\lambda I_{n} & 0 & 0
\end{array}\right]
$$


is also a strong linearization.

Following this example, we now present a general procedure to construct the pencil $\lambda M_{1}+M_{0}$ in the Chebyshev pencil (3.22):

Step 1 Choose natural numbers $\epsilon$ and $\mu$ such that

$$
\epsilon+\mu+1=\operatorname{deg}(P(\lambda)) .
$$

Step 2 Via Lemma 3.5, rewrite $P(\lambda)$ as

$$
P(\lambda)=\sum_{i=0}^{\mu} \sum_{j=0}^{\epsilon}\left(\lambda A_{i j}+B_{i j}\right) U_{i}(\lambda) T_{j}(\lambda) .
$$

Step 3 Set

$$
M_{1}(r, s):=B_{\mu+1-r, \epsilon+1-s} \quad \text { and } \quad M_{0}(r, s):=A_{\mu+1-r, \epsilon+1-s}
$$

for $r=1, \ldots, \mu+1$ and $s=1, \ldots, \epsilon+1$.

Equation (3.23) then guarantees that the Chebyshev pencil (3.22) is a strong linearization of $P(\lambda)$.

REMARK 2. One may wonder if strong linearizations for the matrix polynomial (3.21) can be constructed from Chebyshev pencils (3.1) with $i=j=1$. The following example answers this question in the affirmative. The pencil

$\left[\begin{array}{cccc|ccc}4 \lambda P_{7}+2 P_{6} & P_{5}-P_{7} & 0 & P_{3} & I_{m} & 0 & 0 \\ P_{5}-P_{7} & 2 P_{4} & 0 & 0 & -2 \lambda I_{m} & I_{m} & 0 \\ 0 & 0 & 2 P_{2} & 0 & -I_{m} & -2 \lambda I_{m} & -I_{m} \\ 0 & 0 & P_{1}-P_{5}-P_{7} & P_{0}-P_{2}-P_{4}-P_{6} & 0 & I_{m} & -\lambda I_{m} \\ \hline I_{n} & -2 \lambda I_{n} & I_{n} & 0 & 0 & 0 & 0 \\ 0 & I_{n} & -2 \lambda I_{n} & I_{n} & 0 & 0 & 0 \\ 0 & 0 & I_{n} & -\lambda I_{n} & 0 & 0 & 0\end{array}\right]$

is a strong linearization of the matrix polynomial $P(\lambda)=\sum_{k=0}^{7} P_{k} T_{k}(\lambda)$ since

$$
\begin{array}{r}
{\left[\begin{array}{l}
T_{3}(\lambda) I_{m} \\
T_{2}(\lambda) I_{m} \\
T_{1}(\lambda) I_{m} \\
T_{0}(\lambda) I_{m}
\end{array}\right]^{T}\left[\begin{array}{cccc}
4 \lambda P_{7}+2 P_{6} & P_{5}-P_{7} & 0 & P_{3} \\
P_{5}-P_{7} & 2 P_{4} & 0 & 0 \\
0 & 0 & 2 P_{2} & 0 \\
0 & 0 & P_{1}-P_{5}-P_{7} & P_{0}-P_{2}-P_{4}-P_{6}
\end{array}\right]\left[\begin{array}{l}
T_{3}(\lambda) I_{n} \\
T_{2}(\lambda) I_{n} \\
T_{1}(\lambda) I_{n} \\
T_{0}(\lambda) I_{n}
\end{array}\right]} \\
=P(\lambda),
\end{array}
$$

as may be easily be verified using the formula $2 T_{r}(\lambda) T_{s}(\lambda)=T_{r+s}(\lambda)+T_{|r-s|}(\lambda)$. However, notice that some of the block entries of this linearization are computed via linear combinations involving a large number of the coefficients of $P(\lambda)$, and thus may suffer from cancellation errors. As the degree of $P(\lambda)$ gets larger, the worst this problem becomes. We have not found a way to circumvent this phenomenon. For this reason, in this work we will not pursue the approach of constructing linearizations from Chebyshev pencils (3.1) with $i=j=1$.

3.4.2. Chebyshev polynomials of the second kind. We now focus on matrix polynomials of the form

$$
P(\lambda)=P_{d} U_{d}(\lambda)+P_{d-1} U_{d-1}(\lambda)+\cdots+P_{1} U_{1}(\lambda)+P_{0} U_{0}(\lambda) .
$$


In this situation, the strong linearizations introduced in this section are based on Chebyshev pencils (3.1) with $i=2$ and $j=2$, that is, we consider pencils of the form

$$
\mathcal{L}(\lambda)=\left[\begin{array}{c|c}
\lambda M_{1}+M_{0} & C_{\mu}^{(2)}(\lambda)^{T} \otimes I_{m} \\
\hline C_{\epsilon}^{(2)}(\lambda) \otimes I_{n} & 0
\end{array}\right] .
$$

As in the previous section, we consider the matrices $M_{0}$ and $M_{1}$ in (3.27) as $(\mu+$ 1) $\times(\epsilon+1)$ block matrices $\left[M_{0}(i, j)\right]$ and $\left[M_{1}(i, j)\right]$, where $M_{0}(i, j), M_{1}(i, j) \in \mathbb{F}^{m \times n}$. Recall from Theorem 3.4 that the Chebyshev pencil (3.27) is a strong linearization of a matrix polynomial (3.26) if $\epsilon+\mu+1=d$ and

$$
\begin{aligned}
P(\lambda)= & \left(\Pi_{\mu}^{(2)}(\lambda)^{T} \otimes I_{m}\right)\left(\lambda M_{1}+M_{0}\right)\left(\Pi_{\epsilon}^{(2)}(\lambda) \otimes I_{n}\right)= \\
& \sum_{r=1}^{\mu+1} \sum_{s=1}^{\epsilon+1}\left(\lambda M_{1}(r, s)+M_{0}(r, s)\right) U_{\mu+1-r}(\lambda) U_{\epsilon+1-s}(\lambda) .
\end{aligned}
$$

The equation above shows that finding expressions of Chebyshev polynomials of the second kind in terms of products of the form $U_{\ell_{1}}(\lambda) U_{\ell_{2}}(\lambda)$ is of fundamental importance. Lemma 3.6 gives one way in which this may be done.

Lemma 3.6. The Chebyshev polynomials of the second kind $\left\{U_{j}(\lambda)\right\}$ satisfy the recurrence relations

$$
\begin{aligned}
& U_{r+\ell}(\lambda)=U_{r}(\lambda) U_{\ell}(\lambda)-U_{r-1}(\lambda) U_{\ell-1}(\lambda), \quad \text { and } \\
& U_{r+\ell+1}(\lambda)=2 \lambda U_{r}(\lambda) U_{\ell}(\lambda)-U_{r}(\lambda) U_{\ell-1}(\lambda)-U_{r-1}(\lambda) U_{\ell}(\lambda),
\end{aligned}
$$

where we set $U_{-1}(\lambda):=0$.

Proof. The proof is immediate from the multiplication formula (3.24).

Analogously to the developments in the previous section, from Lemma (3.6) and Equation (3.28) we obtain the following procedure to construct the pencil $\lambda M_{1}+M_{0}$ so that the Chebyshev pencil (3.27) is a strong linearization of the matrix polynomial $P(\lambda)$ in $(3.26)$ :

Step 1 Choose natural numbers $\epsilon$ and $\mu$ such that

$$
\epsilon+\mu+1=\operatorname{deg}(P(\lambda)) .
$$

Step 2 Via Lemma 3.6, rewrite $P(\lambda)$ as

$$
P(\lambda)=\sum_{i=0}^{\mu} \sum_{j=0}^{\epsilon}\left(\lambda A_{i j}+B_{i j}\right) U_{i}(\lambda) U_{j}(\lambda) .
$$

Step 3 Set

$$
M_{1}(r, s):=B_{\mu+1-r, \epsilon+1-s} \quad \text { and } \quad M_{0}(r, s):=A_{\mu+1-r, \epsilon+1-s},
$$

for $r=1, \ldots, \mu+1$ and $s=1, \ldots, \epsilon+1$.

Equation (3.28) then guarantees that the Chebyshev pencil (3.27) is a strong linearization of $P(\lambda)$.

In Example 2 we illustrate the previous procedure for constructing strong linearizations of a matrix polynomial with a small degree $(d=6)$. 
ExAmple 2. Consider the $m \times n$ matrix polynomial $P(\lambda)=\sum_{k=0}^{6} P_{k} U_{k}(\lambda)$, and the Chebyshev pencil (3.27) with $\epsilon=2$ and $\mu=3$. We may write

$$
\begin{aligned}
P(\lambda)= & P_{6}\left(2 \lambda U_{3}(\lambda) U_{2}(\lambda)-U_{3}(\lambda) U_{1}(\lambda)-U_{2}(\lambda) U_{2}(\lambda)\right)+ \\
& P_{5}\left(2 \lambda U_{2}(\lambda) U_{2}(\lambda)-U_{2}(\lambda) U_{1}(\lambda)-U_{1}(\lambda) U_{2}(\lambda)\right)+ \\
& P_{4}\left(2 \lambda U_{1}(\lambda) U_{2}(\lambda)-U_{1}(\lambda) U_{1}(\lambda)-U_{0}(\lambda) U_{2}(\lambda)\right)+ \\
& P_{3} U_{3}(\lambda) U_{0}(\lambda)+P_{2} U_{2}(\lambda) U_{0}(\lambda)+P_{1} U_{1}(\lambda) U_{0}(\lambda)+P_{0} U_{0}(\lambda) U_{0}(\lambda) .
\end{aligned}
$$

The procedure to construct Chebyshev pencils then gives

$$
\left[\begin{array}{ccc|ccc}
2 \lambda P_{6} & -P_{6} & P_{3} & I_{m} & 0 & 0 \\
2 \lambda P_{5}-P_{6} & -P_{5} & P_{2} & -2 \lambda I_{m} & I_{m} & 0 \\
2 \lambda P_{4}-P_{5} & -P_{4} & P_{1} & I_{m} & -2 \lambda I_{m} & I_{m} \\
-P_{4} & 0 & P_{0} & 0 & I_{m} & -2 \lambda I_{m} \\
\hline I_{n} & -2 \lambda I_{n} & I_{n} & 0 & 0 & 0 \\
0 & I_{n} & -2 \lambda I_{n} & 0 & 0 & 0
\end{array}\right]
$$

is a strong linearization of $P(\lambda)$. We could have also written $P(\lambda)$ as

$$
\begin{aligned}
P(\lambda)= & P_{6}\left(2 \lambda U_{3}(\lambda) U_{2}(\lambda)-U_{3}(\lambda) U_{1}(\lambda)-U_{2}(\lambda) U_{2}(\lambda)\right)+ \\
& P_{5}\left(U_{3}(\lambda) U_{2}(\lambda)-U_{2}(\lambda) U_{1}(\lambda)\right)+ \\
& P_{4}\left(2 \lambda U_{1}(\lambda) U_{2}(\lambda)-U_{1}(\lambda) U_{1}(\lambda)-U_{0}(\lambda) U_{2}(\lambda)\right)+ \\
& P_{3}\left(U_{1}(\lambda) U_{2}(\lambda)-U_{0}(\lambda) U_{1}(\lambda)\right)+P_{3} U_{3}(\lambda) U_{0}(\lambda)- \\
& P_{3}\left(2 \lambda U_{2}(\lambda) U_{0}(\lambda)-U_{1}(\lambda) U_{0}(\lambda)\right)+ \\
& P_{2} U_{2}(\lambda) U_{1}(\lambda)+2 \lambda P_{1} U_{0}(\lambda) U_{0}(\lambda)+P_{0} U_{0}(\lambda) U_{0}(\lambda) .
\end{aligned}
$$

The procedure to construct Chebyshev pencils then gives

$$
\left[\begin{array}{ccc|ccc}
2 \lambda P_{6}-P_{5} & -P_{6} & P_{3} & I_{m} & 0 & 0 \\
-P_{6} & -P_{5} & -2 \lambda P_{3}+P_{2} & -2 \lambda I_{m} & I_{m} & 0 \\
2 \lambda P_{4}+P_{3} & -P_{4} & P_{3} & I_{m} & -2 \lambda I_{m} & I_{m} \\
-P_{4} & -P_{3} & 2 \lambda P_{1}+P_{0} & 0 & I_{m} & -2 \lambda I_{m} \\
\hline I_{n} & -2 \lambda I_{n} & I_{n} & 0 & 0 & 0 \\
0 & I_{n} & -2 \lambda I_{n} & 0 & 0 & 0
\end{array}\right]
$$

which is also a strong linearization of $P(\lambda)$.

We conclude this section presenting two block symmetric linearizations for $n \times n$ matrix polynomials of odd degree, as in (3.26). In this case, let us define $s:=(d-1) / 2$, and consider Chebyshev pencils (as in (3.27)) with $\epsilon=\mu=s$. The first of these block symmetric pencils is based on writing $P(\lambda)$ as

$$
\begin{aligned}
P(\lambda)= & \sum_{j=0}^{s} P_{d-2 j}\left(2 \lambda U_{s-j}(\lambda) U_{s-j}(\lambda)-U_{s-j}(\lambda) U_{s-j-1}(\lambda)-\right. \\
& \left.U_{s-j-1}(\lambda) U_{s-j}(\lambda)\right)+ \\
& \sum_{j=0}^{s} P_{d-1-2 j}\left(U_{s-j}(\lambda) U_{s-j}(\lambda)-U_{s-j-1}(\lambda) U_{s-j-1}(\lambda)\right) .
\end{aligned}
$$


Applying the procedure in the previous section to this expansion for $P(\lambda)$, we construct $\lambda M_{1}+M_{0}$ as

$$
\left[\begin{array}{ccccc}
2 \lambda P_{d}+P_{d-1} & -P_{d} & & & \\
-P_{d} & 2 \lambda P_{d-2}+P_{d-3}-P_{d-1} & -P_{d-2} & & \\
& -P_{d-2} & 2 \lambda P_{d-4}+P_{d-5}-P_{d-3} & \ddots & \\
& \ddots & \ddots & -P_{3} \\
& & & -P_{3} & 2 \lambda P_{1}+P_{0}-P_{2}
\end{array}\right]
$$

The second symmetric pencil is based on writing $P(\lambda)$ as

$$
\begin{aligned}
P(\lambda)= & P_{d}\left(2 \lambda U_{s}(\lambda) U_{s}(\lambda)-U_{s-1}(\lambda) U_{s}(\lambda)-U_{s}(\lambda) U_{s-1}(\lambda)+\right. \\
& \sum_{j=0}^{s-2} P_{d-1-2 j}\left(U_{s-2 j}(\lambda) U_{s-2 j}(\lambda)-U_{s-2 j-1}(\lambda) U_{s-2 j-1}(\lambda)\right)+ \\
& \sum_{j=0}^{s-1} \frac{1}{2} P_{d-2-2 j}\left(U_{s-j}(\lambda) U_{s-j-1}(\lambda)-U_{s-j-1}(\lambda) U_{s-j-2}(\lambda)\right)+ \\
& \sum_{j=0}^{s-1} \frac{1}{2} P_{d-2-2 j}\left(U_{s-j-1}(\lambda) U_{s-j}(\lambda)-U_{s-j-2}(\lambda) U_{s-j-1}(\lambda)\right) .
\end{aligned}
$$

Applying the procedure to this expansion for $P(\lambda)$ again, we construct $\lambda M_{1}+M_{0}$ as

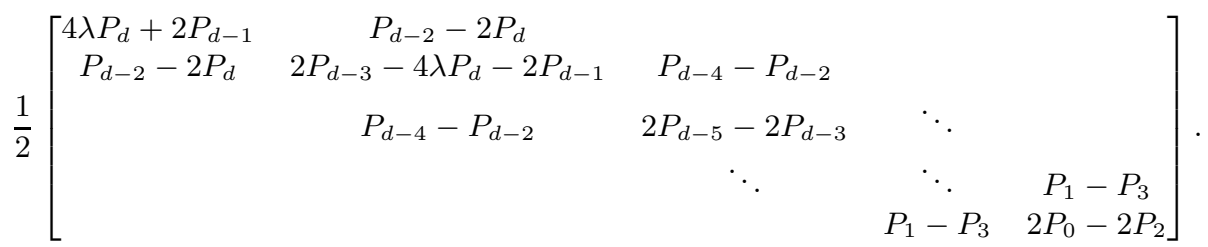

Chebyshev pencils constructed using the block symmetric pencils $\lambda M_{1}+M_{0}$ above are block symmetric strong linearizations of $P(\lambda)$. This implies that these pencils are symmetric strong linearizations whenever the matrix polynomial $P(\lambda)$ is symmetric (that is, whenever $P_{i}^{T}=P_{i}$, for $\left.i=0,1, \ldots, d\right)$. These linearizations are illustrated in the following example.

EXAMPLE 3. Consider the symmetric $n \times n$ matrix polynomial $P(\lambda)=$ $\sum_{k=0}^{5} P_{k} U_{k}(\lambda)$. Then, the Chebyshev pencils

$\left[\begin{array}{ccc|cc}2 \lambda P_{5}+P_{4} & -P_{5} & 0 & I_{n} & 0 \\ -P_{5} & 2 \lambda P_{3}+P_{2}-P_{4} & -P_{3} & -2 \lambda I_{n} & I_{n} \\ 0 & -P_{3} & 2 \lambda P_{1}+P_{0}-P_{2} & I_{n} & -2 \lambda I_{n} \\ \hline I_{n} & -2 \lambda I_{n} & I_{n} & 0 & 0 \\ 0 & I_{n} & -2 \lambda I_{n} & 0 & 0\end{array}\right]$,

and

$$
\left[\begin{array}{ccc|cc}
2 \lambda P_{5}+P_{4} & P_{3} / 2-P_{5} & 0 & I_{n} & 0 \\
P_{3} / 2-P_{5} & P_{2}-P_{4} & P_{1} / 2-P_{3} / 2 & -2 \lambda I_{n} & I_{n} \\
0 & P_{1} / 2-P_{3} / 2 & P_{0}-P_{2} & I_{n} & -2 \lambda I_{n} \\
\hline I_{n} & -2 \lambda I_{n} & I_{n} & 0 & 0 \\
0 & I_{n} & -2 \lambda I_{n} & 0 & 0
\end{array}\right]
$$


are symmetric strong linearizations of $P(\lambda)$.

REMARK 3. Here, we outline a method for constructing strong linearizations of the matrix polynomial (3.21) using the techniques introduced in this section for the matrix polynomial (3.26). First, notice that the following well-known relations between Chebyshev polynomials of the first- and second-kind hold:

$$
\begin{aligned}
& T_{j}(\lambda)=\left(U_{j}(\lambda)-U_{j-2}(\lambda)\right) / 2 \quad \text { with } j \geq 1, \\
& T_{j}(\lambda)=\lambda U_{j-1}(\lambda)-U_{j-2}(\lambda) \quad \text { with } j \geq 1, \quad \text { and } \\
& T_{j}(\lambda)=U_{j}(\lambda)-\lambda U_{j-1}(\lambda),
\end{aligned}
$$

where we set $U_{-1}:=0$ and $U_{-2}:=0$, and thus, we may write $P(\lambda)$ as

$$
P(\lambda)=\sum_{k=0}^{d-1}\left(\lambda F_{k}+E_{k}\right) U_{k}(\lambda) .
$$

more, using Lemma 3.6, we then may write $P(\lambda)$ as

$$
P(\lambda)=\sum_{i=0}^{\mu} \sum_{j=0}^{\epsilon}\left(\lambda A_{i j}+B_{i j}\right) U_{i}(\lambda) U_{j}(\lambda),
$$

with $\epsilon+\mu=\operatorname{deg}(P(\lambda))-1$. Hence, we can construct strong linearizations of $P(\lambda)$ based on a Chebyshev pencils as in (3.27). For example, consider the $n \times n$ matrix polynomial $P(\lambda)=\sum_{k=0}^{d=5} P_{k} T_{k}(\lambda)$. We may write

$$
\begin{aligned}
P(\lambda)= & \left(\lambda P_{5}+P_{4} / 2\right) U_{4}(\lambda)+\left(P_{3} / 2-P_{5}\right) U_{3}(\lambda)+\left(P_{2} / 2-P_{4} / 2\right) U_{2}(\lambda)+ \\
& \left(P_{1} / 2-P_{3} / 2\right) U_{1}(\lambda)+\left(P_{0}-P_{2} / 2\right) U_{0}(\lambda) .
\end{aligned}
$$

Then, using Lemma 3.6 we may write

$$
\begin{aligned}
P(\lambda)= & \left(\lambda P_{5}+P_{4} / 2\right)\left(U_{2}(\lambda) U_{2}(\lambda)-U_{1}(\lambda) U_{1}(\lambda)\right)+ \\
& \left(P_{3} / 2-P_{5}\right)\left(2 \lambda U_{1}(\lambda) U_{1}(\lambda)-U_{1}(\lambda) U_{0}(\lambda)-U_{0}(\lambda) U_{1}(\lambda)\right)+ \\
& \left(P_{2} / 2-P_{4} / 2\right)\left(U_{2}(\lambda) U_{0}(\lambda) / 2+U_{0}(\lambda) U_{2}(\lambda) / 2\right)+ \\
& \left(P_{1} / 2-P_{3} / 2\right) 2 \lambda U_{0}(\lambda) U_{0}(\lambda)+\left(P_{0}-P_{2} / 2\right) U_{0}(\lambda) U_{0}(\lambda) .
\end{aligned}
$$

Finally, using this last expansion for $P(\lambda)$, it may be easily checked that the pencil

$$
\left[\begin{array}{ccc|cc}
\lambda P_{5}+P_{4} / 2 & 0 & \left(P_{2}-P_{4}\right) / 4 & I_{n} & 0 \\
0 & \lambda\left(P_{3}-3 P_{5}\right)-P_{4} / 2 & -P_{3} / 2+P_{5} & -2 \lambda I_{n} & I_{n} \\
\left(P_{2}-P_{4}\right) / 4 & -P_{3} / 2+P_{5} & \lambda\left(P_{1}-P_{3}\right)+P_{0}-P_{2} / 2 & 0 & -2 \lambda I_{n} \\
\hline I_{n} & -2 \lambda I_{n} & I_{n} & 0 & 0 \\
0 & I_{n} & -2 \lambda I_{n} & 0 & 0
\end{array}\right]
$$

is a strong linearization of $P(\lambda)$. Additionally, notice that this linearization is symmetric whenever $P(\lambda)$ is symmetric.

4. One-sided factorizations, eigenvector formulas, and recovery procedures for minimal indices and minimal bases. In this section, we show that any Chebyshev pencil and its reversal satisfy simple one-sided factorizations. Using these factorizations, we show that the eigenvectors (when $P(\lambda)$ is regular), minimal indices, and minimal bases (when $P(\lambda)$ is singular) of $P(\lambda)$ are related to those of any Chebyshev pencil in a simple way. This, in turn, will allow us to easily recover eigenvectors, minimal indices, and minimal bases of $P(\lambda)$ from those of Chebyshev pencils. 
4.1. One-sided factorizations. Let us start by considering the unimodular matrix polynomials constructed in the proof of Theorem 3.4 to show that $\mathcal{L}(\lambda)$ is a linearization of $P(\lambda)$. These are

$$
\begin{aligned}
U(\lambda)= & {\left[\begin{array}{ccc}
I_{\mu m} & 0 & -Z(\lambda) \\
0 & 0 & I_{\epsilon n} \\
0 & I_{m} & -Y(\lambda)
\end{array}\right]\left[\begin{array}{cc}
M_{\mu}(\lambda)^{T} \otimes I_{m} & 0 \\
\Pi_{\mu}^{(j)}(\lambda)^{T} \otimes I_{m} & 0 \\
0 & I_{\epsilon n}
\end{array}\right]=} \\
& {\left[\begin{array}{cc}
M_{\mu}(\lambda)^{T} \otimes I_{m} & -Z(\lambda) \\
0 & I_{\epsilon n} \\
\Pi_{\mu}^{(j)}(\lambda)^{T} \otimes I_{m} & -Y(\lambda)
\end{array}\right], }
\end{aligned}
$$

and

$$
\begin{aligned}
& V(\lambda)=\left[\begin{array}{ccc}
M_{\epsilon}(\lambda) \otimes I_{n} & \Pi_{\epsilon}^{(i)} \otimes I_{n} & 0 \\
0 & 0 & I_{\mu m}
\end{array}\right]\left[\begin{array}{ccc}
0 & I_{\epsilon n} & 0 \\
0 & 0 & I_{n} \\
I_{\mu m} & 0 & -X(\lambda)
\end{array}\right]= \\
& {\left[\begin{array}{ccc}
0 & M_{\epsilon}(\lambda) \otimes I_{n} & \Pi_{\epsilon}^{(i)} \otimes I_{n} \\
I_{\mu m} & 0 & -X(\lambda)
\end{array}\right]}
\end{aligned}
$$

where the matrix polynomials $X(\lambda), Y(\lambda)$, and $Z(\lambda)$ are defined in (3.19). Additionally, let the last block column and the last block row of $V(\lambda)$ and $U(\lambda)$ be denoted by $H(\lambda)$ and $G(\lambda)$, respectively. Explicitly, these are

$$
H(\lambda)=\left[\begin{array}{c}
\Pi_{\epsilon}^{(i)}(\lambda) \otimes I_{n} \\
-\left(M_{\mu}(\lambda)^{T} \otimes I_{m}\right)\left(\lambda M_{1}+M_{0}\right)\left(\Pi_{\epsilon}^{(i)}(\lambda) \otimes I_{n}\right)
\end{array}\right],
$$

and

$$
G(\lambda)=\left[\Pi_{\mu}^{(j)}(\lambda)^{T} \otimes I_{m} \quad-\left(\Pi_{\mu}^{(j)}(\lambda) \otimes I_{m}\right)\left(\lambda M_{1}+M_{0}\right)\left(M_{\epsilon}(\lambda) \otimes I_{n}\right)\right] .
$$

In Theorem 4.1, we show that any Chebyshev pencil (3.1) satisfies simple leftsided and right-sided factorizations.

TheOREM 4.1. Let $\mathcal{L}(\lambda)$ be the Chebyshev pencil $(3.1), P(\lambda)$ be the matrix polynomial in (2.1), and $H(\lambda)$ and $G(\lambda)$ be the matrix polynomials in (4.1) and (4.2). The following relations hold:

$$
\mathcal{L}(\lambda) H(\lambda)=e_{\mu+1} \otimes P(\lambda) \quad \text { and } \quad G(\lambda) \mathcal{L}(\lambda)=e_{\epsilon+1}^{T} \otimes P(\lambda)
$$

where $e_{\ell}$ denotes the $\ell$ th column of the $d \times d$ identity matrix $(d:=\epsilon+\mu+1)$.

Proof. From the linearization equation (2.4), we have that

$$
\mathcal{L}(\lambda) H(\lambda)=U(\lambda)^{-1}\left(e_{d} \otimes P(\lambda)\right) \quad \text { and } \quad G(\lambda) \mathcal{L}(\lambda)=\left(e_{d}^{T} \otimes P(\lambda)\right) V(\lambda)^{-1} .
$$

We thus need to establish that $U(\lambda)^{-1}\left(e_{d} \otimes P(\lambda)\right)=e_{\mu+1} \otimes P(\lambda)$ and $\left(e_{d}^{T} \otimes P(\lambda)\right) V(\lambda)^{-1}$ $=e_{\epsilon+1}^{T} \otimes P(\lambda)$. Indeed, we have

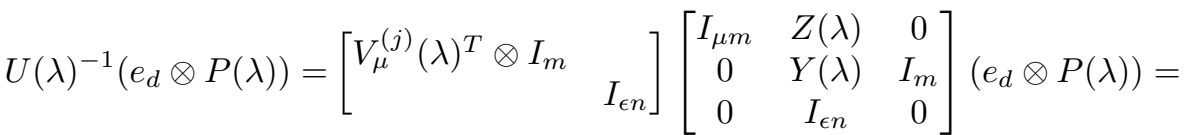

$$
\begin{aligned}
& {\left[\begin{array}{cc}
V_{\mu}^{(j)}(\lambda) \otimes I_{m} & \\
& I_{\epsilon n}
\end{array}\right]\left(e_{\mu+1} \otimes P(\lambda)\right)=e_{\mu+1} \otimes P(\lambda) \text {, }}
\end{aligned}
$$


and

$$
\begin{aligned}
& \left(e_{d}^{T} \otimes P(\lambda)\right) V(\lambda)^{-1}=\left(e_{d}^{T} \otimes P(\lambda)\right)\left[\begin{array}{ccc}
0 & X(\lambda) & I_{\mu m} \\
I_{\epsilon n} & 0 & 0 \\
0 & I_{n} & 0
\end{array}\right]\left[\begin{array}{ll}
V_{\epsilon}^{(j)}(\lambda) & \\
& I_{\mu m}
\end{array}\right]= \\
& \left(e_{\epsilon+1}^{T} \otimes P(\lambda)\right)\left[\begin{array}{ll}
V_{\epsilon}^{(j)}(\lambda) & \\
& I_{\mu m}
\end{array}\right]=e_{\epsilon+1}^{T} \otimes P(\lambda) .
\end{aligned}
$$

Let us now consider the unimodular matrix polynomials

$$
\begin{aligned}
\widehat{U}(\lambda)= & {\left[\begin{array}{ccc}
0 & 0 & I_{\epsilon n} \\
0 & I_{\mu m} & -\widehat{Z}(\lambda) \\
I_{m} & 0 & -\widehat{Y}(\lambda)
\end{array}\right]\left[\begin{array}{cc}
\operatorname{rev}_{\mu} \Pi_{\mu}^{(j)}(\lambda)^{T} \otimes I_{m} & 0 \\
N_{\mu}^{(j)}(\lambda)^{T} \otimes I_{m} & 0 \\
0 & I_{\epsilon}
\end{array}\right]=} \\
& {\left[\begin{array}{cc}
0 & I_{\epsilon n} \\
N_{\mu}^{(j)}(\lambda)^{T} \otimes I_{m} & -\widehat{Z}(\lambda) \\
\operatorname{rev}_{\mu} \Pi_{\mu}^{(j)}(\lambda)^{T} \otimes I_{m} & -\widehat{Y}(\lambda)
\end{array}\right], }
\end{aligned}
$$

and

$$
\begin{aligned}
& \widehat{V}(\lambda)=\left[\begin{array}{ccc}
\operatorname{rev}_{\epsilon} \Pi_{\epsilon}^{(i)}(\lambda) \otimes I_{n} & N_{\epsilon}^{(i)}(\lambda) \otimes I_{n} & 0 \\
0 & 0 & I_{\mu m}
\end{array}\right]\left[\begin{array}{ccc}
0 & 0 & I_{n} \\
I_{\epsilon n} & 0 & 0 \\
0 & I_{\mu m} & -\widehat{X}(\lambda)
\end{array}\right]= \\
& {\left[\begin{array}{ccc}
N_{\epsilon}^{(i)}(\lambda) \otimes I_{n} & 0 & \operatorname{rev}_{\epsilon} \Pi_{\epsilon}^{(i)}(\lambda) \otimes I_{n} \\
0 & I_{\mu m} & -\widehat{X}(\lambda)
\end{array}\right]}
\end{aligned}
$$

constructed in the proof of Theorem 3.4 to show that $\operatorname{rev}_{1} \mathcal{L}(\lambda)$ is a linearization of $\operatorname{rev}_{d} P(\lambda)$, where the matrix polynomials $\widehat{X}(\lambda), \widehat{Y}(\lambda)$, and $\widehat{Z}(\lambda)$ are defined in (3.20). The last block column and the last block row of $\widehat{V}(\lambda)$ and $\widehat{U}(\lambda)$ are given by

$$
\widehat{H}(\lambda)=\left[\begin{array}{c}
\operatorname{rev}_{\epsilon} \Pi_{\epsilon}^{(i)}(\lambda) \otimes I_{n} \\
-\left(N_{\mu}^{(j)}(\lambda)^{T} \otimes I_{m}\right)\left(\lambda M_{0}+M_{1}\right)\left(\operatorname{rev}_{\epsilon} \Pi_{\epsilon}^{(i)}(\lambda) \otimes I_{n}\right)
\end{array}\right],
$$

and

$$
\widehat{G}(\lambda)=\left[\operatorname{rev}_{\mu} \Pi_{\mu}^{(j)}(\lambda)^{T} \otimes I_{m} \quad-\left(\operatorname{rev}_{\mu} \Pi_{\mu}^{(j)}(\lambda)^{T} \otimes I_{m}\right)\left(\lambda M_{0}+M_{1}\right)\left(N_{\epsilon}^{(i)}(\lambda) \otimes I_{n}\right)\right],
$$

respectively. Finally, let us define the following vector

$$
\widehat{\omega}_{k}^{(i)}=\left[\begin{array}{lllllll}
\omega_{k}^{(i)} & \cdots & \omega_{1}^{(i)} & \omega_{0}^{(i)} & 0 & \cdots & 0
\end{array}\right]^{T} \in \mathbb{C}^{d \times 1}, \quad \text { with } i \in\{1,2\},
$$

where the scalars $\omega_{0}^{(i)}, \omega_{1}^{(i)}, \ldots, \omega_{k}^{(i)}$ are defined in Lemma 3.2.

In Theorem 4.2, we show that the reversal of any Chebyshev pencil also satisfies simple left-sided and right-sided factorizations.

TheOREM 4.2. Let $\mathcal{L}(\lambda)$ be the Chebyshev pencil $(3.1), P(\lambda)$ be the matrix polynomial in (2.1), and $\widehat{H}(\lambda)$ and $\widehat{G}(\lambda)$ be the matrix polynomials in (4.4) and (4.5). The following relations hold:

$$
\operatorname{rev}_{1} \mathcal{L}(\lambda) \widehat{H}(\lambda)=\widehat{\omega}_{\mu}^{(j)} \otimes \operatorname{rev}_{d} P(\lambda) \quad \text { and } \quad \widehat{G}(\lambda) \operatorname{rev}_{1} \mathcal{L}(\lambda)=\left(\widehat{\omega}_{\epsilon}^{(i)}\right)^{T} \otimes \operatorname{rev}_{d} P(\lambda)
$$


Proof. From the linearization equation (2.5), we have that $\operatorname{rev}_{1} \mathcal{L}(\lambda) \widehat{H}(\lambda)=\widehat{U}(\lambda)^{-1}\left(e_{d} \otimes \operatorname{rev}_{d} P(\lambda)\right) \quad$ and $\quad \widehat{G}(\lambda) \operatorname{rev}_{1} \mathcal{L}(\lambda)=\left(e_{d}^{T} \otimes \operatorname{rev}_{d} P(\lambda)\right) \widehat{V}(\lambda)^{-1}$

We thus need to establish that $\widehat{U}(\lambda)^{-1}\left(e_{d} \otimes \operatorname{rev}_{d} P(\lambda)\right)=\widehat{\omega}_{\mu}^{(j)} \otimes \operatorname{rev}_{d} P(\lambda)$ and $\left(e_{d}^{T} \otimes\right.$ $\left.\operatorname{rev}_{d} P(\lambda)\right) \widehat{V}(\lambda)^{-1}=\left(\widehat{\omega}_{\epsilon}^{(i)}\right)^{T} \otimes \operatorname{rev}_{d} P(\lambda)$. Indeed, we have

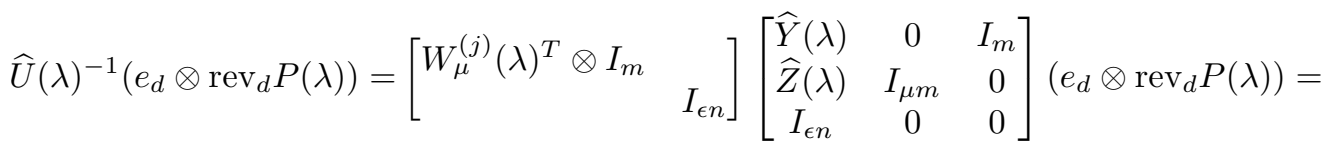

$$
\begin{aligned}
& {\left[\begin{array}{ll}
W_{\mu}^{(j)}(\lambda)^{T} \otimes I_{m} & \\
& I_{\epsilon n}
\end{array}\right]\left(e_{1} \otimes \operatorname{rev}_{d} P(\lambda)\right)=\widehat{\omega}_{\mu}^{(j)} \otimes \operatorname{rev} P(\lambda),}
\end{aligned}
$$

and

$$
\begin{aligned}
& \left(e_{d}^{T} \otimes \operatorname{rev}_{d} P(\lambda)\right) \widehat{V}(\lambda)^{-1}=\left(e_{d}^{T} \otimes \operatorname{rev}_{d} P(\lambda)\right)\left[\begin{array}{ccc}
0 & I_{\epsilon n} & 0 \\
\widehat{X}(\lambda) & 0 & I_{\mu m} \\
I_{n} & 0 & 0
\end{array}\right]\left[\begin{array}{lll}
W_{\epsilon}^{(i)}(\lambda)^{T} \otimes I_{n} & \\
& & I_{\epsilon n}
\end{array}\right]=
\end{aligned}
$$

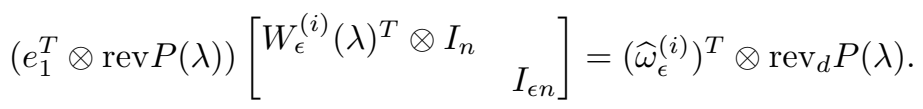

4.2. Minimal indices and bases. In this section, we assume that the matrix polynomial $P(\lambda)$ is singular. The following theorem shows that the minimal indices and minimal bases of $P(\lambda)$ and those of any of its Chebyshev linearizations are closely related.

Theorem 4.3. Let $P(\lambda)$ be a matrix polynomial as in $(2.1), \mathcal{L}(\lambda)$ be a Chebyshev linearization of $P(\lambda)$ as in (3.1), and $H(\lambda)$ and $G(\lambda)$ be the matrix polynomials in (4.1) and (4.2), respectively.

(a1) Suppose that $\left\{z_{1}(\lambda), z_{2}(\lambda), \ldots, z_{p}(\lambda)\right\}$ is any right minimal basis of $\mathcal{L}(\lambda)$, with vectors partitioned into blocks conformable to the blocks of $\mathcal{L}(\lambda)$, and let $x_{\ell}(\lambda)$ be the $(\epsilon+1)$ th block of $z_{\ell}(\lambda)$, for $\ell=1,2, \ldots, p$. Then $\left\{x_{1}(\lambda), x_{2}(\lambda), \ldots, x_{p}(\lambda)\right\}$ is a right minimal basis of $P(\lambda)$.

(a2) Suppose that $\left\{x_{1}(\lambda), x_{2}(\lambda), \ldots, x_{p}(\lambda)\right\}$ is any right minimal basis of $P(\lambda)$. Then $\left\{H(\lambda) x_{1}(\lambda), H(\lambda) x_{2}(\lambda), \ldots, H(\lambda) x_{p}(\lambda)\right\}$ is a right minimal basis of $\mathcal{L}(\lambda)$.

(a3) If $0 \leq \epsilon_{1} \leq \epsilon_{2} \leq \cdots \leq \epsilon_{p}$ are the right minimal indices of $P(\lambda)$, then

$$
0 \leq \epsilon_{1}+\epsilon \leq \epsilon_{2}+\epsilon \leq \cdots \leq \epsilon_{p}+\epsilon
$$

are the right minimal indices of $\mathcal{L}(\lambda)$.

(b1) Suppose that $\left\{w_{1}(\lambda)^{T}, w_{2}(\lambda)^{T}, \ldots, w_{q}(\lambda)^{T}\right\}$ is any left minimal basis of $\mathcal{L}(\lambda)$, with vectors partitioned into blocks conformable to the blocks of $\mathcal{L}(\lambda)$, and let $y_{\ell}(\lambda)^{T}$ be the $(\mu+1)$ th block of $w_{\ell}(\lambda)^{T}$, for $\ell=1,2, \ldots, q$. Then $\left\{y_{1}(\lambda)^{T}\right.$, $\left.y_{2}(\lambda)^{T}, \ldots, y_{q}(\lambda)^{T}\right\}$ is a left minimal basis of $P(\lambda)$.

(b2) Suppose that $\left\{y_{1}(\lambda), y_{2}(\lambda), \ldots, y_{q}(\lambda)\right\}$ is any left minimal basis of $P(\lambda)$. Then $\left\{y_{1}(\lambda)^{T} G(\lambda), y_{2}(\lambda)^{T} G(\lambda), \ldots, y_{q}(\lambda)^{T} G(\lambda)\right\}$ is a left minimal basis of $\mathcal{L}(\lambda)$. 
(b3) If $0 \leq \mu_{1} \leq \mu_{2} \leq \cdots \leq \mu_{q}$ are the left minimal indices of $P(\lambda)$, then

$$
0 \leq \mu_{1}+\mu \leq \mu_{2}+\mu \leq \cdots \leq \mu_{q}+\mu
$$

are the left minimal indices of $\mathcal{L}(\lambda)$.

Proof. We prove parts (a1), (a2) and (a3), since the proofs for parts (b1), (b2) and (b3) are similar. It follows from (4.3) that $x(\lambda) \in \mathcal{N}_{r}(P)$ if and only if $H(\lambda) x(\lambda) \in$ $\mathcal{N}_{r}(\mathcal{L})$. As a consequence of the structure of $H(\lambda)$ in $(4.1)$, we also see that $x(\lambda)$ is a vector polynomial if and only if $z=H(\lambda) x(\lambda)$ is a vector polynomial. Additionally, the structure of $H(\lambda)$ implies that a list of vectors $x_{1}(\lambda), \ldots, x_{j}(\lambda) \in \mathcal{N}_{r}(P)$ is linearly independent if and only if the list $z_{1}(\lambda)=H(\lambda) x_{1}(\lambda), \ldots, z_{j}(\lambda)=H(\lambda) x_{j}(\lambda)$ is linearly independent. Therefore, the linear map $z(\lambda)=H(\lambda) x(\lambda)$ induces a one-toone correspondence between vector polynomial bases of $\mathcal{N}_{r}(\mathcal{L})$ and vector polynomial bases of $\mathcal{N}_{r}(P)$.

To complete the argument, we first show that for a nonzero vector polynomial $x(\lambda) \in \mathcal{N}_{r}(P)$ it holds that $\operatorname{deg}(H(\lambda) x(\lambda))=\operatorname{deg}(x(\lambda))+\epsilon$. To see why the previous uniform degree-shifting property holds, we first note that there are only two different types of blocks in $H(\lambda) x(\lambda)$, namely, $T_{\ell}(\lambda) x(\lambda)$ with $0 \leq \ell \leq \epsilon$, and

$$
-\left(M_{\mu}(\lambda)^{T} \otimes I_{m}\right)\left(\lambda M_{1}+M_{0}\right)\left(\Pi_{\epsilon}^{(i)}(\lambda) \otimes I_{n}\right) x(\lambda)=-X(\lambda) x(\lambda) .
$$

Clearly, the maximum degree among all blocks of the form $T_{\ell}(\lambda) x(\lambda)$ is $\operatorname{deg}(x(\lambda))+$ $\epsilon$, attained only in the topmost block of $H(\lambda) x(\lambda)$. We now need to prove that $\operatorname{deg}(X(\lambda) x(\lambda)) \leq \operatorname{deg}(x(\lambda))+\epsilon$.

Since $x(\lambda) \in \mathcal{N}_{r}(P)$, pre- and post-multiplying (4.3) by $\lambda^{\mu}$ and $x(\lambda)$, respectively, we must have that

$$
\mathcal{L}(\lambda)\left[\begin{array}{c}
\lambda^{\mu} \Pi_{\epsilon}^{(i)}(\lambda) \otimes x(\lambda) \\
-\lambda^{\mu} X(\lambda) x(\lambda)
\end{array}\right]=e_{\mu+1} \otimes\left(\lambda^{\mu} P(\lambda) x(\lambda)\right)=0 .
$$

Furthermore, taking the $d$-reverse in (4.6) we similarly obtain that

$$
\begin{aligned}
& \mathcal{L}(\lambda)\left[\begin{array}{c}
\lambda^{\mu} \Pi_{\epsilon}^{(i)}(\lambda) \otimes x(\lambda) \\
-\left(\operatorname{rev}_{\mu-1} N_{\mu}^{(j)}(\lambda)^{T} \otimes I_{m}\right)\left(\lambda M_{1}+M_{0}\right)\left(\Pi_{\epsilon}^{(i)}(\lambda) \otimes x(\lambda)\right)
\end{array}\right]= \\
& \widehat{\omega}_{\mu}^{(j)} \otimes(P(\lambda) x(\lambda))=0,
\end{aligned}
$$

where we have used the fact that $\epsilon+\mu=d-1$ and that $\operatorname{deg}\left(N_{\mu}^{(j)}(\lambda)\right) \leq \mu-1$ (see Lemma 3.3). Subtracting the two previous equations yields

$$
\mathcal{L}(\lambda)\left[\begin{array}{c}
0 \\
-\lambda^{\mu} X(\lambda) x(\lambda)+\left(\operatorname{rev}_{\mu-1} N_{\mu}^{(j)}(\lambda)^{T} \otimes I_{m}\right)\left(\lambda M_{1}+M_{0}\right)\left(\Pi_{\epsilon}^{(i)}(\lambda) \otimes x(\lambda)\right)
\end{array}\right]=0,
$$

which implies that

$$
\begin{aligned}
&\left(C_{\mu}^{(j)}(\lambda)^{T} \otimes I_{m}\right)\left(-\lambda^{\mu} X(\lambda) x(\lambda)+\right. \\
&\left.\left(\operatorname{rev}_{\mu-1} N_{\mu}^{(j)}(\lambda)^{T} \otimes I_{m}\right)\left(\lambda M_{1}+M_{0}\right)\left(\Pi_{\epsilon}^{(i)}(\lambda) \otimes I_{n}\right) x(\lambda)\right)=0 .
\end{aligned}
$$

Since $C_{\mu}^{(j)}(\lambda)^{T} \otimes I_{m}$ has full column rank, we may conclude that

$$
\lambda^{\mu} X(\lambda) x(\lambda)=\left(\operatorname{rev}_{\mu-1} N_{\mu}^{(j)}(\lambda)^{T} \otimes I_{m}\right)\left(\lambda M_{1}+M_{0}\right)\left(\Pi_{\epsilon}^{(i)}(\lambda) \otimes x(\lambda)\right) .
$$

Inspecting the degrees in the equation above we finally obtain that $\operatorname{deg}(X(\lambda) x(\lambda)) \leq$ $\operatorname{deg}(x(\lambda))+\epsilon$ 
4.3. Eigenvectors. In this section, we assume that the matrix polynomial $P(\lambda)$ is regular. In the following theorem, we show that the eigenvectors of $P(\lambda)$ and those of any of its Chebyshev linearizations are closely related. In addition, we show how to recover the eigenvectors of $P(\lambda)$ from those of its linearizations without any computational cost. The existence of an eigenvector recovery procedure is essential for a linearization to be relevant for applications. We start by considering eigenvectors associated with finite eigenvalues.

TheOREM 4.4. Let $P(\lambda)$ be a regular matrix polynomial as in $(2.1), \mathcal{L}(\lambda)$ be a Chebyshev linearization as in (3.1), and $H(\lambda)$ and $G(\lambda)$ be the matrix polynomials in (4.1) and (4.2), respectively.

(a1) If $z \in \mathbb{F}^{n d \times 1}$ is a right eigenvector of $\mathcal{L}(\lambda)$ with finite eigenvalue $\lambda$, then the $(\epsilon+1)$ th block of $z$ is a right eigenvector of $P(\lambda)$ with finite eigenvalue $\lambda$.

(a2) If $x \in \mathbb{F}^{n \times 1}$ is a right eigenvector of $P(\lambda)$ with finite eigenvalue $\lambda$, then $H(\lambda) x$ is a right eigenvector of $\mathcal{L}(\lambda)$ with eigenvalue $\lambda$.

(b1) If $w^{*} \in \mathbb{F}^{1 \times n d}$ is a left eigenvector of $\mathcal{L}(\lambda)$ with finite eigenvalue $\lambda$, then the $(\mu+1)$ th block of $w^{*}$ is a left eigenvector of $P(\lambda)$ with finite eigenvalue $\lambda$.

(b2) If $y^{*} \in \mathbb{F}^{1 \times n}$ is a left eigenvector of $P(\lambda)$ with finite eigenvalue $\lambda$, then $y^{*} G(\lambda)$ is a left eigenvector of $\mathcal{L}(\lambda)$ with eigenvalue $\lambda$.

Proof. We focus on the right eigenvectors, since the results for the left eigenvectors follow similar arguments. From (4.3) we immediately obtain that $x$ is a right eigenvector of $P(\lambda)$ with finite eigenvalue $\lambda$ if and only if $z=H(\lambda) x$ is a right eigenvector of $\mathcal{L}(\lambda)$ with finite eigenvalue $\lambda$. The $(\epsilon+1)$ th block entry of $H(\lambda)$ is equal to the identity matrix (recall that $T_{0}(\lambda)=1$ ), and thus, we obtain that the right eigenvectors of $P(\lambda)$ may be recovered from the $(\epsilon+1)$ th blocks of those of $\mathcal{L}(\lambda)$.

We now consider the eigenvectors associated with eigenvalues at infinity.

THEOREM 4.5. Let $P(\lambda)$ be a regular matrix polynomial as in $(2.1), \mathcal{L}(\lambda)$ be a Chebyshev linearization as in (3.1), and $\widehat{H}(\lambda)$ and $\widehat{G}(\lambda)$ be the matrix polynomials in (4.4) and (4.5), respectively.

(a1) If $z \in \mathbb{F}^{n d \times 1}$ is a right eigenvector of $\mathcal{L}(\lambda)$ for the eigenvalue $\infty$, then $(-1)^{\epsilon} \sum_{\ell=0}^{\epsilon} \omega_{\ell}^{(i)} z_{\ell}$ is a right eigenvector of $P(\lambda)$ for the eigenvalue $\infty$.

(a2) If $x \in \mathbb{F}^{n d \times 1}$ is a right eigenvector of $P(\lambda)$ for the $\infty$ eigenvalue, then $\widehat{H}(0) x$ is a right eigenvector of $\mathcal{L}(\lambda)$ for the eigenvalue $\infty$.

(b1) If $w^{*} \in \mathbb{F}^{1 \times n d}$ is a left eigenvector of $\mathcal{L}(\lambda)$ for the eigenvalue $\infty$, then $(-1)^{\mu} \sum_{\ell=0}^{\mu} \omega_{\ell}^{(j)} w_{\ell}^{*}$ is a left eigenvector of $P(\lambda)$ for the $\infty$ eigenvalue.

(b2) If $y^{*} \in \mathbb{F}^{1 \times n}$ is a left eigenvector of $P(\lambda)$ for the $\infty$ eigenvalue, then $y^{*} \widehat{G}(0)$ is a left eigenvector of $\mathcal{L}(\lambda)$ for the eigenvalue $\infty$.

Proof. Again, we focus only on right eigenvectors. Recall that a regular matrix polynomial $P(\lambda)$ has an infinite eigenvalue if and only if $\operatorname{rev}_{d} P(\lambda)$ has an eigenvalue at zero, and the corresponding right eigenvectors of $P(\lambda)$ at the eigenvalue $\infty$ are just the right null vectors of $\operatorname{rev}_{d} P(0)$. From (4.6) we immediately obtain that $x$ is a right eigenvector of $\operatorname{rev}_{d} P(\lambda)$ with eigenvalue $\lambda=0$ if and only if $z=\widehat{H}(0) x$ is a right eigenvector of $\operatorname{rev}_{1} \mathcal{L}(\lambda)$ with eigenvalue $\lambda=0$. Finally, using (3.15) we obtain

$$
(-1)^{\epsilon} \sum_{\ell=0}^{\epsilon} \omega_{\ell}^{(i)} z_{\ell}=(-1)^{\epsilon} \sum_{\ell=0}^{\epsilon} \omega_{\ell}^{(i)} \operatorname{rev}_{k} T_{k}(\lambda) x=x
$$


if $i=1$, and

$$
(-1)^{\epsilon} \sum_{\ell=0}^{\epsilon} \omega_{\ell}^{(i)} z_{\ell}=(-1)^{\epsilon} \sum_{\ell=0}^{\epsilon} \omega_{\ell}^{(i)} \operatorname{rev}_{k} U_{k}(\lambda) x=x
$$

if $i=2$.

5. Conclusion and future work. In this article, we have proposed a new approach for constructing strong linearizations for polynomials expressed in Chebyshev bases, which we call Chebyshev pencils. These Chebyshev pencils have block antisymmetric structure, which affords us significant flexibility to distribute the coefficients of the polynomial within the diagonal block of the linearization. The structure provides a clear separation between the parts containing the coefficients of the polynomial and the parts containing the recurrence relations of the Chebyshev bases. A novelty of such a separation is that this allows us to construct linearizations having a mixture of products of first- and second-kind Chebyshev polynomials. We suspect that this is not the end of the story, and that further generalizations of this idea will give rise to new linearizations based on the mixing of other polynomial bases. We have established the strong linearization property for the Chebyshev pencils by embedding certain parts of the pencil (and their reversals) in unimodular matrices, giving us a uniform way to construct linearizations of polynomial matrices. From these unimodular matrices, we are then able to linearize a polynomial in one of the two Chebyshev bases by factoring the Chebyshev polynomials in terms of products of lower degree Chebyshev polynomials. We have also given the relationships between the eigenvectors, minimal indices, and minimal bases of the linearization and those of the matrix polynomial. These procedures give simple ways in which to recover the complete eigenstructure of a polynomial matrix from the eigenstructure of the Chebyshev pencil. Our approach is applied only to matrix polynomials expressed in Chebyshev bases. However, it is easily seen that the general approach is applicable to other polynomial bases, and we expect that generalizations for other bases will be seen in the coming years.

Furthermore, besides their intrinsic matrix polynomial theoretical interest, the results obtained in Section 4 about one-sided factorizations find applications in numerical analysis, e.g., for conditioning [17] and backward error analysis [16]. In addition, it is well known that different linearizations of the same matrix polynomial can have widely varying eigenvalue condition numbers or produce widely varying backward errors. For this reason, the analysis of the influence of the linearization process presented in this work on the accuracy and stability of the computed solution of a polynomial eigenvalue problem will be the subject of future work.

\section{REFERENCES}

[1] M. Abramowitz And I. A. Stegun, Handbook of Mathematical Functions: with Formulas, Graphs, and Mathematical Tables, Courier Dover Publications, 1972.

[2] A. Amiraslani, R. M. Corless, and P. Lancaster, Linearization of matrix polynomials expressed in polynomial bases, IMA J. Numer. Anal., 29 (2009), pp. 141-157.

[3] Stephen BARnett, A companion matrix analogue for orthogonal polynomials, Linear Algebra Appl., 12 (1975), pp. 197-202.

[4] - Leverrier's algorithm for orthogonal polynomial bases, Linear Algebra Appl., 236 (1996), pp. $245-263$.

[5] T. Beelen And P. VAn Dooren, A pencil approach for embedding a polynomial matrix into a unimodular matrix, SIAM J. Matrix Anal. Appl., 9 (1988), pp. 77-89. 
[6] M. I. Bueno, F. M Dopico, S. Furtado, And M. Rychnovsky, Large vector spaces of blocksymmetric strong linearizations of matrix polynomials, Linear Algebra Appl., 477 (2015), pp. $165-210$.

[7] F. De Terán, F. M. Dopico, And D. S. Mackey, Fiedler companion linearizations and the recovery of minimal indices, SIAM J. Matrix Anal. Appl., 31 (2010), pp. 2181-2204.

[8] - Palindromic companion forms for matrix polynomials of odd degree, J. Comput. Appl. Math., 236 (2011), pp. 1464-1480.

[9] - Spectral equivalence of matrix polynomials and the index sum theorem, Linear Algebra Appl., 459 (2014), pp. 264-333.

[10] F. De Terán, F. M. Dopico, And J. PÉrez, Backward stability of polynomial root-finding using Fiedler companion matrices, IMA J. Numer. Anal., (2015), pp. pp. 1-41.

[11] F. M. Dopico, P. W. Lawrence, J. PÉrez, And P. VAn Dooren, Fiedler-like linearizations, In preparation, (2015).

[12] C. EfFEnBERger AND D. KREssner, Chebyshev interpolation for nonlinear eigenvalue problems, BIT, 52 (2012), pp. 933-951.

[13] D. K. Faddeev and I. S. Sominskil, Problems in higher algebra, Gostekhizdat, Moscow, $2 / 5$ ed., $1949 / 1954$.

[14] G. D. Forney, JR., Minimal bases of rational vector spaces, with applications to multivariable linear systems, SIAM J. Control, 13 (1975), pp. 493-520.

[15] I. J. Good, The colleague matrix, a Chebyshev analogue of the companion matrix, Q. J. Math., 12 (1961), pp. 61-68.

[16] N. J. Higham, R.-C.. Li, And F. Tisseur, Backward error of polynomial eigenproblems solved by linearization, SIAM J. Matrix Anal. Appl., 29 (2007), pp. 1218-1241.

[17] N. J. Higham, D. S. Mackey, And F. Tisseur, The conditioning of linearizations of matrix polynomials, SIAM J. Matrix Anal. Appl., 28 (2006), pp. 1005-1028.

[18] Peter LAncaster, Linearization of regular matrix polynomials, Electron. J. Linear Algebra, 17 (2008), pp. 21-27.

[19] P. W. LaWrence, M. VAN BARel, And P. VAN Dooren, Backward error analysis of polynomial eigenvalue problems solved by linearization, SIAM J. Matrix Anal. Appl., (2015). To appear.

[20] U. J. J. LEVERRIER, Sur les variations séculaires des éléments des orbites pour les sept planètes principales, J. de Math., 1 (1840), pp. 220-254.

[21] D. S. Mackey, N. Mackey, C. Mehl, and V. Mehrmann, Structured polynomial eigenvalue problems: Good vibrations from good linearizations, SIAM J. Matrix Anal. Appl., 28 (2006), pp. 1029-1051.

$[22] \longrightarrow$, Vector spaces of linearizations for matrix polynomials, SIAM J. Matrix Anal. Appl., 28 (2006), pp. 971-1004.

[23] D. S. Mackey, N. Mackey, And F. Tisseur, Polynomial eigenvalue problems: Theory, computation, and structure, in Numerical Algebra, Matrix Theory, Differential-Algebraic Equations and Control Theory, Peter Benner, Matthias Bollhöfer, Daniel Kressner, Christian Mehl, and Tatjana Stykel, eds., Springer International Publishing, 2015, pp. 319-348.

[24] D. S. MACKey AND V. Perović, Linearizations of matrix polynomials in Bernstein basis, MIMS EPrint 2015.90, Manchester Institute for Mathematical Sciences, The University of Manchester, UK, 2014.

[25] — Linearizations of matrix polynomials in Newton basis, In preparation, (2015).

[26] V. Mehrmann and H. Voss, Nonlinear eigenvalue problems: A challenge for modern eigenvalue methods, GAMM-Mitteilungen, 27 (2004), pp. 121-152.

[27] Y. NAKatsukasa And V. Noferini, On the stability of computing polynomial roots via confederate linearizations, To appear in Math. Comp., (2015).

[28] Y. Nakatsukasa, V. Noferini, and A. Townsend, Vector spaces of linearizations for matrix polynomials: a bivariate polynomial approach, MIMS EPrint 2012.118, Manchester Institute for Mathematical Sciences, The University of Manchester, UK, 2015.

[29] V. Noferini and J. PÉREz, Chebyshev-Fiedler pencils, MIMS EPrint 2015.90, Manchester Institute for Mathematical Sciences, The University of Manchester, UK, 2015.

[30] — Chebyshev rootfinding via computing eigenvalues of colleague matrices: when is it stable?, MIMS EPrint 2015.24, Manchester Institute for Mathematical Sciences, The University of Manchester, UK, 2015.

[31] L. N. Trefethen, Approximation theory and approximation practice, SIAM, 2013.

[32] L. N. Trefethen And Others, Chebfun version 5, The Chebfun Development Team http://www.maths.ox.ac.uk/chebfun/, (2014). 